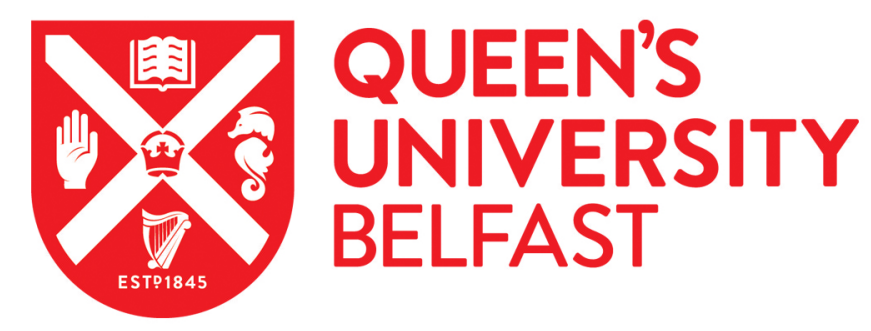

\title{
Type lax supernovae as a few-parameter family
}

Barna, B., Szalai, T., Kerzendorf, W. E., Kromer, M., Sim, S. A., Magee, M. R., \& Leibundgut, B. (2018). Type lax supernovae as a few-parameter family. Monthly Notices of the Royal Astronomical Society, 480(3), 36093627. https://doi.org/10.1093/mnras/sty2065

Published in:

Monthly Notices of the Royal Astronomical Society

Document Version:

Publisher's PDF, also known as Version of record

Queen's University Belfast - Research Portal:

Link to publication record in Queen's University Belfast Research Portal

Publisher rights

(C) 2018 The Authors. Published by Oxford University Press on behalf of the Royal Astronomical Society. This work is made available online in accordance with the publisher's policies. Please refer to any applicable terms of use of the publisher.

\section{General rights}

Copyright for the publications made accessible via the Queen's University Belfast Research Portal is retained by the author(s) and / or other copyright owners and it is a condition of accessing these publications that users recognise and abide by the legal requirements associated with these rights.

Take down policy

The Research Portal is Queen's institutional repository that provides access to Queen's research output. Every effort has been made to ensure that content in the Research Portal does not infringe any person's rights, or applicable UK laws. If you discover content in the Research Portal that you believe breaches copyright or violates any law, please contact openaccess@qub.ac.uk. 


\title{
Type Iax supernovae as a few-parameter family
}

\author{
Barnabás Barna, ${ }^{1,2 \star}$ Tamás Szalai, ${ }^{1}$ Wolfgang E. Kerzendorf, ${ }^{2}$ Markus Kromer,,${ }^{3,4}$ \\ Stuart A. Sim, ${ }^{5}$ Mark R. Magee ${ }^{5}$ and Bruno Leibundgut ${ }^{2}$ \\ ${ }^{1}$ Department of Optics and Quantum Electronics, University of Szeged, Dom ter 9, 6720 Szeged, Hungary \\ ${ }^{2}$ European Southern Observatory, Karl-Schwarzschild-Strasse 2, 85748 Garching bei München, Germany \\ ${ }^{3}$ Heidelberger Institut für Theoretische Studien, Schloss-Wolfsbrunnenweg 35, D-69118 Heidelberg, Germany \\ ${ }^{4}$ Zentrum für Astronomie der Universität Heidelberg, Institut für Theoretische Astrophysik, Philosophenweg 12, D-69120 Heidelberg, Germany \\ ${ }^{5}$ Astrophysics Research Centre, School of Mathematics and Physics, Queen's University Belfast, Belfast BT7 1NN, UK
}

Accepted 2018 July 28. Received 2018 July 13; in original form 2018 March 19

\begin{abstract}
We present the direct spectroscopic modelling of five Type Iax supernovae (SNe) with the 1D Monte Carlo radiative transfer code TARDIS. The abundance tomography technique is used to map the chemical structure and physical properties of the $\mathrm{SN}$ atmospheres. By fitting multiple spectral epochs with self-consistent ejecta models, we can constrain the location of some elements within the ejecta. The synthetic spectra of the best-fitting models can reproduce the flux continuum and the main absorption features in the whole sample. We find that the mass fractions of iron-group elements and intermediate-mass elements show a decreasing trend towards the outer regions of the atmospheres when we use density profiles similar to those of deflagration models in the literature. Oxygen is the only element that could be dominant at higher velocities. The stratified abundance structure contradicts the well-mixed chemical profiles predicted by pure deflagration models. Based on the derived densities and abundances, a template model atmosphere is created for the SN Iax class and compared with the observed spectra. The free parameters are the scaling of the density profile, the velocity shift of the abundance template, and the peak luminosity. The results of this research support the idea that all SNe Iax can be described by a similar internal structure, which argues for a common origin of this class of explosions.
\end{abstract}

Key words: line: formation - line: identification - radiative transfer; supernovae: general.

\section{INTRODUCTION}

Most Type Ia supernovae ( $\mathrm{SNe}$ Ia) form a one-parameter family often referred to as 'Branch-normal' (Branch et al. 2006). The amount of synthesized ${ }^{56} \mathrm{Ni}$ determines their peak luminosity, which correlates with the shape of their light curves, making these explosions standardizable candles and ideal for distance measurements on cosmological scales. However, certain thermonuclear white dwarf (WD) explosions do not follow this correlation, and some of their other observable properties are usually also peculiar compared with those of normal SNe Ia. A special group of these objects, named after the prototypical member of them (Li et al. 2003), are '2002cx'-like $\mathrm{SNe}$, or, as they have become known following Foley et al. (2013), Type Iax SNe.

The characteristic observational properties of SNe Iax are the low peak luminosities, which extend over a wide range from the extremely faint $\mathrm{SN} 2008$ ha $\left(M_{\mathrm{V} \text {,peak }} \sim-14\right.$ mag; Valenti et al.
2009) up to the relatively luminous SN 2011ay ( $M_{\mathrm{V} \text {,peak }} \sim-18.4$ mag; Foley et al. 2013; Szalai et al. 2015). Their photospheric velocities are also significantly lower than those of normal Type Ia SNe, typically falling between 5000 and $8000 \mathrm{~km} \mathrm{~s}^{-1}$ at maximum light (Foley et al. 2013), but objects with expansion velocities of $2500 \mathrm{~km} \mathrm{~s}^{-1}$ have also been observed (Foley et al. 2009; Stritzinger et al. 2014). Although a general, but not tight, correlation can be observed between the luminosity and velocity values, some SNe Iax do not fit into this sequence, such as SN 2009ku (Narayan et al. 2011; Foley et al. 2013) and SN 2014ck (Tomasella et al. 2016).

Beyond the wide scale of peak luminosities, the light curves show further diversity. The rise times of SNe Iax are shorter than the typical values for SNe Ia $(18.0 \pm 2.0 \mathrm{~d}$ in the $B$ band, as found by Ganeshalingham, Li \& Filippenko 2011); however, the typically faster decline rates do not seem to correlate with the peak luminosities. The near-infrared light curves do not display a second peak, suggesting strong mixing in the ejecta of SNe Iax (Jha et al. 2006; Phillips et al. 2007).

The early spectra of SNe Iax are dominated by lines of irongroup elements (IGEs). Spectral lines of intermediate-mass ele- 
ments (IME), for example SiII and Ca II, are always present, but their strength is far from that observed in normal SNe Ia. Highvelocity features have not been observed in the spectra of any $\mathrm{SNe}$ Iax. At later epochs, both permitted absorption features and forbidden emission lines can be found at optical wavelengths, in contrast to the case for spectra of other thermonuclear explosions (Jha et al. 2006).

The study of SNe Iax has the potential to answer questions related to progenitor systems of thermonuclear SNe. While no progenitor system of normal Type Ia SNe has ever been discovered, McCully et al. (2014) reported a possible source coincident with Type Iax SN $2012 Z$ on a pre-explosion image of the Hubble Space Telescope (HST). The detected luminous blue star could be a potential He star donor to the exploding white dwarf. The existence of such progenitor systems, suggested first by Foley et al. (2013), is supported by detailed binary evolution calculations (Wang, Justham \& Han 2013). However, Liu et al. (2015) noted that this kind of system is unlikely to be the progenitor of the majority of SNe Iax, because the long delay time of the single degenerate Chandrasekhar mass $\left(M_{\mathrm{Ch}}\right)$ models does not seem to explain the observed number of Type Iax SNe.

The above-mentioned observables (i.e. low peak luminosity and photospheric velocity; strong mixing) and the spectral footprints of chemical elements together indicate that deflagrations play a major role in the explosion of Type Iax SNe. The subsonic explosions of carbon-oxygen (CO) $M_{\mathrm{Ch}}$ WDs leaving bound remnants (also referred to as 'failed' $\mathrm{SNe}$ ) have been investigated using 3D hydrodynamical simulations (Jordan et al. 2012b; Kromer et al. 2013). According to these and other studies (Long et al. 2016; Magee et al. 2016), the synthetic observables of weak pure deflagrations are able to broadly reproduce both the photometric and the spectroscopic properties of SNe Iax.

Fink et al. (2014) presented a set of 3D hydrodynamical calculations using a multispot ignition approach to scale the strength of the deflagration. The peak luminosities of their models N3def, N5def, N10def and N20def are comparable with those of the more luminous SNe Iax (Foley et al. 2013). The growing number of ignition spots produces more energetic and luminous explosions in accordance with the wide range of the observed luminosities and expansion velocities. In addition, the pure deflagration models of Fink et al. (2014) show highly mixed abundance structures with nearly constant mass fractions for each element.

The main goal of this study is to carry out a comprehensive abundance tomography (Stehle et al. 2005) analysis involving several SNe Iax. The sample of objects is chosen to broadly represent the properties of the class. Similar to in our pilot study for SN 2011ay (Barna et al. 2017), we compare our findings with the results of previous spectral models, as well as with the predictions of the pure deflagration of a Chandrasekhar-mass WD with a bound remnant (the most promising explosion scenario for $\mathrm{SNe}$ Iax).

This paper is structured as follows. In Section 2, we give an overview of the target sample of our study. In Section 3, we show how we use the TARDIS ${ }^{1}$ code (Kerzendorf $\&$ Sim 2014) for abundance tomography in order to simulate the spectral evolution of the objects. In Section 4, we present the results of our spectral modelling, compare the resulting physical picture of the ejecta with other models, and introduce a template for the subclass of SNe Iax. Finally, we summarize our main findings in Section 5.

\footnotetext{
${ }^{1}$ The TARDIS software package is available from: https://zenodo.org/record/
} 1292315 .

\section{SN IAX STUDY SAMPLE}

The adopted technique requires a time sequence. The photosphere assumption of TARDIS (Kerzendorf \& Sim 2014) is robust only within $\sim 30 \mathrm{~d}$ after the explosion (see Section 3), and hence only SNe with at least three spectra within this time range were considered. As a further constraint, we need reliable information on the host galaxy reddening in order to fit the emergent luminosity.

Nearly 60 SNe Iax have been discovered to date, but only a few meet the above-mentioned criteria of our analysis. According to the conditions listed above, five SNe Iax (see Table 1) were chosen from open-access data bases (Yaron \& Gal-Yam 2012; Guillochon et al. 2017). A log of spectroscopic observations used in this study is presented in Table 2.

The reddening value of the Milky Way was adopted from the dust emission map of Schlafly \& Finkbeiner (2011), assuming $R_{V}$ $=3.1$ as the extinction coefficient. All spectra were corrected for the sum of galactic and extra-galactic interstellar reddening based on the extinction model of Fitzpatrick \& Massa (2007).

\subsection{SN 2011ay}

SN 2011 ay is one of the most luminous SNe Iax (Foley et al. 2013), with a peak absolute brightness of $M_{\mathrm{V} \text {,peak }} \sim-18.4$ mag (Szalai et al. 2015). It was discovered by the Katzman Automatic Imaging Telescope (KAIT)/Lick Observatory Supernova Search (LOSS) program in NGC 2318 (Blanchard et al. 2011) at a redshift of $z$ $=0.021$. The host-galaxy reddening was found to be negligible, because no significant $\mathrm{Na} \mathrm{D}$ lines were detected.

The original spectroscopic data of SN 2011ay were published by Silverman et al. (2012), while Szalai et al. (2015) provided a detailed photometric and spectroscopic analysis. In addition, Barna et al. (2017) published an abundance tomography analysis using TARDIS; a revision of these results is part of the current paper.

\subsection{SN $2012 \mathrm{Z}$}

SN $2012 Z$ was also discovered (Cenko et al. 2012) by the KAIT/LOSS program. The peak luminosity of SN 2012Z is comparable to that of SN 2011ay; depending on the adopted distance and host-galaxy reddening, it may be the most luminous SNe Iax (Stritzinger et al. 2014, 2015). The redshift of its host galaxy, NGC 1309 , is $z=0.007$. The host-galaxy reddening was estimated to be $E(B-V)=0.07 \mathrm{mag}$ based on the study of high-resolution $\mathrm{Na} \mathrm{I}$ $\lambda 5890 \lambda 5896$ and $\mathrm{K}_{\mathrm{I}} \lambda 7665 \lambda 7699$ line profiles (Stritzinger et al. $2014,2015)$, and of line strengths of the diffuse interstellar band at $5780 \AA$ A (Phillips et al. 2013).

Spectra of SN 2012Z were published by Yamanaka et al. (2015) and Stritzinger et al. (2014, 2015), who also presented detailed optical spectroscopic analyses.

\subsection{SN 2005hk}

SN 2005hk is one of the best-observed SNe Iax. It was discovered (Burket \& Li 2005) independently by LOSS and SDSS-II in UGC 272 at a redshift of $z=0.012$. The absolute magnitude of SN 2005hk is $-18.08 \mathrm{mag}$ in the $V$ band (Phillips et al. 2007). The host-galaxy reddening was studied via interstellar polarization and $\mathrm{Na} \mathrm{D}$ lines (Chornock et al. 2006) and found to be $E(B-V)=$ 0.09 mag. Different kinds of spectral modelling were carried out by Phillips et al. (2007), Sahu et al. (2008) and Magee et al. (2017); while the authors of the former paper used sYNOw (Fisher 2000), the 
Table 1. Absolute peak magnitudes in the $V$ band (in the $r$ band for SN 2015H), redshifts, distance moduli and reddening values (both in magnitudes) for each $\mathrm{SN}$ in our sample.

\begin{tabular}{lcccccc}
\hline Object & $M_{\text {peak }}$ & $z$ & $\mu$ & $E(B-V)_{\mathrm{MW}}$ & $E(B-V)_{\text {host }}$ & Paper \\
\hline SN 2011ay & $-18.39 \pm 0.18$ & 0.021 & $34.69 \pm 0.15$ & 0.069 & 0.00 & Szalai et al. (2015) \\
SN 2012Z & $-18.50 \pm 0.09$ & 0.007 & $32.59 \pm 0.09$ & 0.036 & & Stritzinger et al. (2014, \\
& & & & & 0.019 & 2015) \\
SN 2005hk & $-18.08 \pm 0.29$ & 0.012 & $33.46 \pm 0.27$ & 0.019 & Phillips et al. (2007) \\
SN 2002cx & $-17.62 \pm 0.35$ & 0.024 & $35.09 \pm 0.32$ & 0.034 & 0.00 & Li et al. (2003) \\
SN 2015H & $-17.27 \pm 0.07$ & 0.012 & $33.91 \pm 0.07$ & 0.048 & Magee et al. (2016) \\
\hline
\end{tabular}

Table 2. Log of the spectra of our sample; the phases are given with respect to the $B$ maximum. The time since explosion $\left(t_{\mathrm{exp}}\right)$ was a fitting parameter for our TARDIS models (see Section 3.1) within $\pm 1.5 \mathrm{~d}$ of their estimated values in the referenced papers.

\begin{tabular}{|c|c|c|c|c|}
\hline$t_{\exp }[\mathrm{d}]$ & Phase [d] & Telescope $\backslash$ Instrument & Wavelength $[\AA]$ & Paper \\
\hline \multicolumn{5}{|c|}{ SN 2011ay } \\
\hline 11.0 & -2.4 & HET $\backslash$ LRS & $4100-10000$ & Szalai et al. (2015) \\
\hline 13.0 & -0.4 & HET $\backslash$ LRS & $4100-10000$ & Szalai et al. (2015) \\
\hline 15.0 & +1.6 & HET $\backslash$ LRS & 4100-10000 & Szalai et al. (2015) \\
\hline 16.0 & +2.6 & Lick $\backslash$ Kast & $3350-9850$ & Silverman et al. (2012) \\
\hline 20.0 & +6.6 & Lick $\backslash$ Kast & $3400-9700$ & Silverman et al. (2012) \\
\hline 23.0 & +9.6 & Lick $\backslash$ Kast & $3400-9700$ & Silverman et al. (2012) \\
\hline 29.0 & +15.6 & Lick $\backslash$ Kast & $3350-10550$ & Silverman et al. (2012) \\
\hline 32.0 & +18.6 & HET $\backslash$ LRS & $4100-10000$ & Szalai et al. (2015) \\
\hline \multicolumn{5}{|c|}{ SN 2012Z } \\
\hline 5.4 & -9.2 & Lick $\backslash$ Kast & $3400-10000$ & $\begin{array}{c}\text { Stritzinger et al. (2014, } \\
2015)\end{array}$ \\
\hline 15.7 & +1.1 & $\mathrm{KAO} \backslash$ LOSA & $4100-7900$ & Yamanaka et al. (2015) \\
\hline 20.3 & +5.7 & FLWO $\backslash$ FAST & $3400-10000$ & $\begin{array}{c}\text { Stritzinger et al. (2014, } \\
2015)\end{array}$ \\
\hline \multicolumn{5}{|c|}{ SN 2005hk } \\
\hline 5.3 & -9.3 & FLWO $\backslash$ FAST & $3500-7400$ & Blondin et al. (2012) \\
\hline 6.3 & -8.3 & Lick $\backslash$ KAST & $3300-10400$ & Phillips et al. (2007) \\
\hline 8.3 & -6.3 & $\mathrm{APO} \backslash \mathrm{DIS}$ & $3600-9600$ & Phillips et al. (2007) \\
\hline 9.5 & -5.1 & Keck $\backslash$ LRIS & $3200-9200$ & Phillips et al. (2007) \\
\hline 10.2 & -4.4 & $\mathrm{APO} \backslash \mathrm{DIS}$ & $3600-9600$ & Phillips et al. (2007) \\
\hline 11.3 & -3.3 & FLWO $\backslash$ FAST & $3500-7400$ & Blondin et al. (2012) \\
\hline 13.3 & -1.3 & FLWO $\backslash$ FAST & $3500-7400$ & Blondin et al. (2012) \\
\hline 18.3 & +3.7 & $\mathrm{MDM} \backslash \mathrm{CDSS}$ & $3900-7300$ & Phillips et al. (2007) \\
\hline
\end{tabular}

Note: ${ }^{*}$ In the case of SN 2015H, the $r$ maximum was used instead of the $B$ maximum.

latter studies employed the Monte Carlo spectrum synthesis codes of Mazzali (2000) and Kerzendorf \& Sim (2014), respectively.

\section{$2.4 \mathrm{SN} 2002 \mathrm{cx}$}

SN 2002cx is the prototype of the Type Iax SN class. It was discovered in the galaxy CGCG 044-035 (Wood-Vasey et al. 2002) at a redshift of $z=0.024$. The peak luminosity of SN 2002cx is $M_{\mathrm{V} \text {,peak }} \sim-17.6 \mathrm{mag}(\mathrm{Li}$ et al. 2003). Near-maximum light spectra were first published by Li et al. (2003), who claimed that the object suffered from no or negligible host-galaxy reddening. Detailed spectral analysis regarding the photospheric phase was presented by Branch et al. (2004), while Jha et al. (2006) presented late-time spectra. 


\subsection{SN 2015H}

SN 2015H was originally discovered (Parker 2015) by the BOSS program in NGC $3464(z=0.012)$, approximately $20 \mathrm{~d}$ after explosion. Thus, no photometric or spectroscopic data were obtained during the pre-maximum phase. An exception is the $r$ band, in which SN 2015H is well observed and shows a peak absolute magnitude of -17.3 mag. The observables were presented by Magee et al. (2016), who modelled one spectrum with TARDIS and compared their findings with the predictions of deflagration models.

\section{METHOD}

We used the 1D radiative spectral synthesis code TARDIS (Kerzendorf \& Sim 2014) to perform the abundance tomography technique, which was originally described by Stehle et al. (2005). As the homologously expanding SN ejecta becomes optically thinner, the velocity of the photosphere decreases and observations can probe deeper into the ejecta. Thus, the analysis of a spectral time series allows the various regions of the $\mathrm{SN}$ ejecta to be mapped.

TARDIS assumes a sharp photosphere emitting a blackbody continuum, above which the model atmosphere is divided into multiple spherically symmetric shells. Indivisible photon packets representing bundles of photons with the same frequency are sent from the bottom of the computation volume, and the code follows their interaction with matter. The chemical abundances and the densities are specified as input parameters in each shell, while additional physical parameters (radiation temperature, ionization and excitation ratios) are computed by the code iteratively.

The temperature of the photosphere is estimated according to the Stefan-Boltzman law from the photospheric velocity and the emergent luminosity. Note that after each TARDIs iteration, the statistics of the Monte Carlo packets are used to recalculate the blackbody temperature at the inner boundary to match the value of the emergent luminosity. The radiative temperature in each radial shell is updated according to the Monte Carlo estimators (Kerzendorf \& Sim 2014), based on the flight histories of the photon packets. Thus, the temperature-dependent conditions are improved iteratively to a more consistent model.

\subsection{Fitting methods}

In this study, we fit all available spectra of each object with a self-consistent ejecta model, changing only the time-dependent parameters. These are the time since explosion, the inner boundary of the modelling volume, the mass fractions of the radioactive isotopes ${ }^{56} \mathrm{Ni}$ and ${ }^{56} \mathrm{Co}$ (which do not result in any additional free parameters in our fitting), and the luminosity, whose change follows the light curve of the SN.

Explosion dates and the derived times since explosion $\left(t_{\exp }\right)$ of the modelled SNe are from earlier studies (see Table 2), but we allow them to vary within their uncertainty range, which is usually $\pm 1.5 \mathrm{~d}$. The same strategy was used for the emergent luminosity $\left(L_{\mathrm{e}}\right)$ parameter, which is calculated from the quasi-bolometric light curves of the earlier studies (except for SN 2015H), with an uncertainty range of 0.1 dex.

The TARDIS input parameter called the 'velocity of the inner boundary' is the bottom border of the computation volume, where a blackbody radiation field is emitted. This description has similarities to the definition of the photosphere, the sharp boundary between the optically thick and thin parts of the atmosphere. Although a real photosphere cannot be expected in SN Iax atmospheres, a mean photosphere is often used in the literature. For these reasons, we refer to the inner-boundary parameter photospheric velocity $\left(v_{\text {phot }}\right)$ hereafter, which is a free fitting parameter in our method.

The upper end of the computation volume is not strictly limited, but the impact of the outer region rapidly decreases with density. The outer boundary is set sufficiently high to $4000 \mathrm{~km} \mathrm{~s}^{-1}$ above $v_{\text {phot }}$ for the first spectral fitting of each object, and we keep it fixed for the later epochs. The total studied velocity range of an object is higher than $4000 \mathrm{~km} \mathrm{~s}^{-1}$, because $v_{\text {phot }}$ decreases with time. Note that the same velocity region, which has negligible impact on the spectrum at later epochs, could be critical at earlier epochs. The model atmosphere is divided into radial velocity shells in steps of $100 \mathrm{~km} \mathrm{~s}^{-1}$. Because we fit the spectra manually, the number of fitting parameters has to be limited. Thus, the mass fractions of the chemical elements are set in a coarser velocity grid, with velocity steps of $500 \mathrm{~km} \mathrm{~s}^{-1}$.

The densities and abundances of our initial models are based on the results of the available hydrodynamical calculations of the pure deflagration scenario, which show the most similarities to the observed properties of SNe Iax. The density and abundance profiles of the model grid presented by Fink et al. (2014) are the starting points in our fitting process, and we modify the corresponding values if the fitting requires it. In practice, the empirical density profiles used in our simulations are exponential functions, namely

$\rho\left(v, t_{\text {exp }}\right)=\rho_{0}\left(\frac{t_{\exp }}{100 \mathrm{~s}}\right)^{-3} \exp \left(-\frac{v}{2300 \mathrm{~km} \mathrm{~s}^{-1}}\right) 64^{\frac{v-v_{\text {cut }}}{v_{\text {cut }}}}$,

with a cut-off towards higher velocities, which can roughly match the basic structure of deflagration models (Fink et al. 2014). These density functions are described by two fitting parameters, namely the value of the central density, $\rho_{\mathrm{n} 0}$ (Barna et al. 2017; Magee et al. 2016), and the location of the cut-off, $v_{\text {cut }}$ (see Table 3 ), where the adopted density profile starts to deviate from the exponential function.

We use only those chemical elements that produce unambiguous spectral features for at least one of the observed epochs. These elements are $\mathrm{C}, \mathrm{O}, \mathrm{Na}, \mathrm{Mg}, \mathrm{Si}, \mathrm{S}, \mathrm{Ca}, \mathrm{Cr}, \mathrm{Fe}, \mathrm{Co}$ and $\mathrm{Ni}$ for our sample; however, the composition can vary from object to object. Elements with no or weak spectral features are not identified during our analysis, which may result in an unknown mass fraction. Based on experience gained from the abundance tomography of SN 2011ay (Barna et al. 2017), a significant fraction of mass is not covered by the above-mentioned elements, especially in the outermost regions. This could be caused by the presence of unidentified hidden elements, or by an incorrect choice of the density profile. In order to avoid any false detections of the elements, we choose oxygen as a 'filler' element to cover the missing mass fraction. Because oxygen forms a strong line only at $\lambda 7771$ a week after the explosion, and which is close to being saturated, increasing its fraction in the model atmosphere does not have a significant impact on the spectrum. This also means that the increasing oxygen abundances (see Section 4.3) towards higher velocities are probably artificial by-products of our fitting method.

In contrast to our previous work (Barna et al. 2017), we use carbon in all of our ejecta models. Because carbon is not formed in the SN atmosphere as a nuclear burning product, but comes from the original matter of the progenitor $\mathrm{WD}$, it is a key element in constraining the progenitor and explosion mechanism of a supernova. CII $\lambda 6580$ has been detected in several SNe Iax (Chornock et al. 2006; Foley et al. 2010; McClelland et al. 2010; Parrent et al. 2011; Thomas et al. 2011), while Foley et al. (2013) have suggested that every SN Iax may show carbon features in their spectra before or 
Table 3. The time since explosion, the emergent luminosity and the photospheric velocity parameters of the best-fit TARDIS models. In the rows of the SNe, the central density values (at a reference time of $100 \mathrm{~s}$ after the explosion) and the locations of the cut-offs in the adopted density functions are shown. The figures show the fitted density profiles of the SNe (solid lines) and the corresponding density functions of the theoretical models (dashed lines)

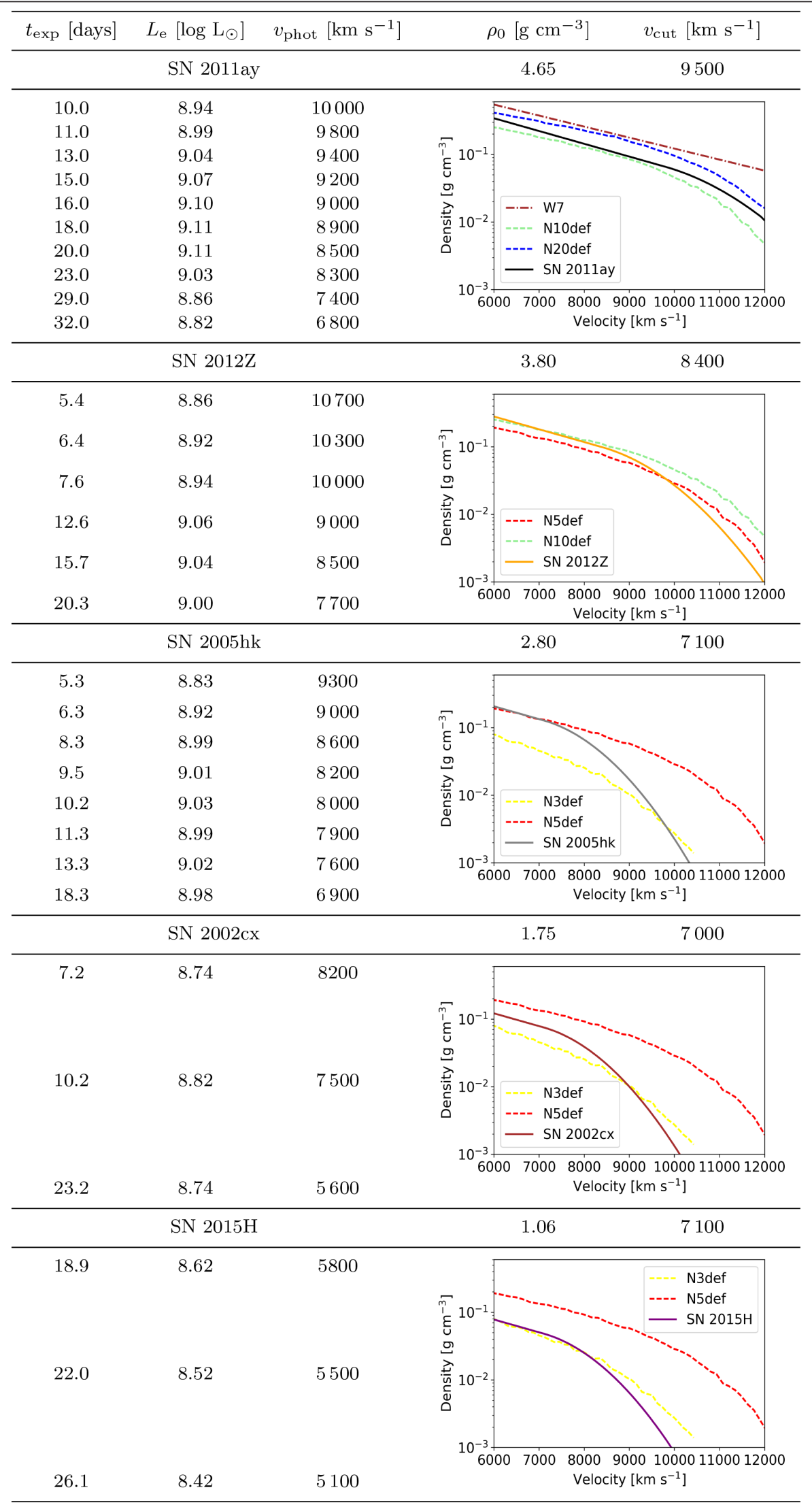


around maximum light. Carbon appears in the theoretical models for SNe Iax with a constant mass fraction of 0.1-0.2, depending on the kinetic energy of the pure deflagration scenario. However, Barna et al. (2017) did not find evidence for the appearance of carbon lines in the spectra of SN 2011ay, and thus carbon was not included in that study. In order to revise the results of Barna et al. (2017) and to extend our investigation of the unburnt material, we have changed our fitting strategy in relation to carbon. Here, we try to discover the upper limit of the element in terms of both location and mass fraction.

To summarize, the free parameters of the individual model of each SN are the time of explosion, the central density $\rho_{\mathrm{n} 0}$, and the location of the density cut-off $v_{\text {cut }}$. The latter two parameters define the density profile. The mass fractions of the chemical elements in each radial shell are also free parameters. Using the same ejecta model for fitting all the spectra of a given SN keeps our modelling approach self-consistent. The parameters $L_{\mathrm{e}}$ and $v_{\text {phot }}$ are fitted for all the available spectra. We explore the parameter space until we find a good visual agreement between the observations and the synthetic spectra.

\section{RESULTS AND DISCUSSION}

Figs 1-5 show that our fitted density and abundance profiles are able to reproduce the flux continuum and the main spectral features in almost all cases.

We list the fitted physical parameters $\left(t_{\exp }, L_{\mathrm{e}}, v_{\text {phot }}\right)$ and the density-profile defining parameters $\left(\rho_{0}\right.$ and $\left.v_{\text {cut }}\right)$ for each epoch of the $\mathrm{SNe}$ in our sample in Table 3 . The fitted density profiles in our individual models show a steeper function than in the cases of the corresponding deflagration models (see Table 3 ). The discrepancies are especially high for SNe 2002ck, 2005hk and 2012Z, where the fitting of the steep flux continuum and of the blue wings of spectral lines requires a stronger cut-off at the higher velocities in the ejecta. In order to illustrate the impact of the cut-off, we compare the effect of the adopted density profile for SN 2012Z with the density function of the N5def model and of the same model without a cut-off at the higher velocities. As can be seen in Fig. 6, a stronger cut-off (lower $v_{\text {cut }}$ value) in the density profile reduces the excessive blueshifts of the spectral lines of IGEs.

All five best-fit models show stratified chemical structures (see Fig. 7), where the mass fractions of almost all the chemical elements change with velocity (for the parameters of the individual abundance profiles, see Tables A1-A5). These models show many similarities in both density and abundance profiles, despite being fitted for different supernovae. Note that these findings are in tension with the deflagration models, which are taken as the initial point for our fitting process (see Section 3.1), because those hydrodynamical calculations predict nearly constant mass fractions for each element. Based on the similar distributions of the chemical elementsin the individual abundance profiles, we create an abundance template for our sample.

In the following, we use the abundance profiles to create a fewparameter description of SN Iax, which we call the template abundance profile (Section 4.1). We compare this template with the general predictions of the pure deflagration models and use it to describe the above-mentioned similar structures of the abundance profiles (see Sections 4.2 and 4.3). Sections 4.4-4.8 describe the unique attributes of each best-fit model, and, if possible, we compare it with other spectroscopic analyses in the literature.

\subsection{Abundance template for the Type Iax sample}

We calculate the mean chemical abundances of the $\mathrm{SNe}$ (except for SN 2015H, see Section 4.8) in each radial shell to create an abundance template model for the whole Type Iax sample. For this purpose, we choose the transition velocity in every abundance profile, where the mass fraction of oxygen is equal to that of radioactive nickel $\left(\mathrm{X}(\mathrm{O})=\mathrm{X}\left({ }^{56} \mathrm{Ni}\right)\right)$ as a reference point. The fitted individual abundance profiles (Fig. 7) are shifted in velocity space to match the the individual transition velocities. Then, we calculate the average mass fractions in each radial shell. The resulting abundance profiles of the chemical elements can be seen in Fig. 8.

The template abundance profile preserves the common characteristics of the individual objects, such as the decreasing abundance of both IMEs and IGEs with increasing velocity or the lack of carbon in the inner regions. However, these features exist at different velocities from object to object in our sample, roughly corresponding to their $v_{\text {phot }}$ values at maximum light. In order to test our hypothesis, we use the abundance template (Fig. 8) with transition velocities of $7000,8500,10000$ and $11000 \mathrm{~km} \mathrm{~s}^{-1}$ for SNe $2015 \mathrm{H}, 2002 \mathrm{cx}$, $2005 \mathrm{hk}$ and 2011ay, respectively, according to the transition velocity of our fits of each object (see Sections 4.4-4.8). We generate synthetic spectra for one epoch of each object and compare them to both the observed spectra and our best-fit models. In order to demonstrate the effect of the same abundance profile at completely different velocities, we choose similar epochs in pairs: approximately $8 \mathrm{~d}$ after the explosion of SN 2002cx and SN 2005hk, and $20 \mathrm{~d}$ in the case of SN 2015H and SN 2011ay. Because our goal is only to test the uniformity of the abundance profile, the luminosities and the times of the explosions, just like the density profiles, remain the same as in the best-fit models.

The effect of the template profile can be seen in Figs 9 and 10, where the quality of the fits weakens only slightly in general. This indicates that we could effectively describe the chemical structure of the SNe in our sample with only one parameter. The fact that the same template abundance profile describes SNe with completely different luminosities increases the possibility that SNe Iax have the same or a similar progenitor scenario. The deflagration-like density profiles that we used, defined with only two free parameters (see Section 3.1), support this assumption. However, at this point, we cannot say that the whole subclass is a one-parameter family like the normal Type Ia SNe, but it seems to be possible that only a few free parameters (velocity shift of the abundance profile, density function) can efficiently describe all SNe Iax.

\subsection{Inner regions of the abundance template}

Fig. 8 shows that the inner layers of the template model atmosphere are dominated by IGEs. The most abundant species is the radioactive ${ }^{56} \mathrm{Ni}$, which reaches a fraction of $0.40-0.50$ in the innermost ejecta and gradually decreases towards the outer regions. The extension of ${ }^{56} \mathrm{Ni}$ and its decay products in our best-fit models is limited by the blue side of their absorption lines at post-maximum epochs. The initial mass fractions of stable iron roughly follow the changes of ${ }^{56} \mathrm{Ni}$, varying between 0.05 and 0.15 . The amount and location of stable iron are constrained by the strong FeIII lines at $\sim 4400$ and $5600 \AA$ in the pre-maximum spectra.

IMEs appear with smaller mass fractions than IGEs at the same velocities. The mass fractions of $\mathrm{Si}$ and $\mathrm{S}$ are well constrained, because of the relatively strong, but not saturated, SirI $\lambda 6355$ and SiIII $\lambda 4588$ lines and the $\mathrm{S}_{\text {II }}$ 'W' absorption feature. The mass fractions of these two elements follow a similar trend between 

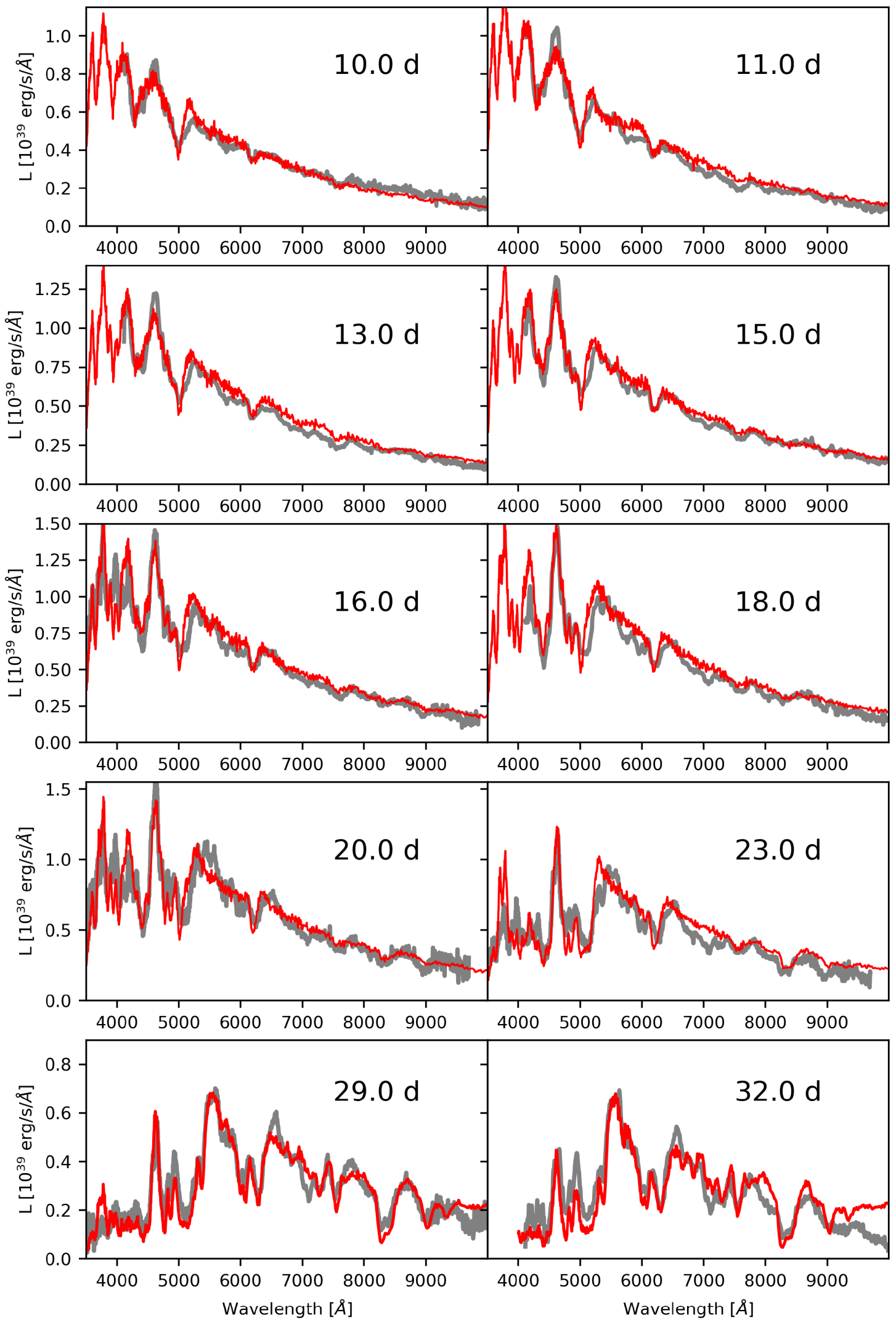

Figure 1. The observed spectra (grey) of SN 2011ay obtained between -3 and $+19 \mathrm{~d}$ with respect to $B$-maximum, compared with our best-fit TARDIs models (red). The numbers show the time since explosion in our best-fit TARDIS models. 

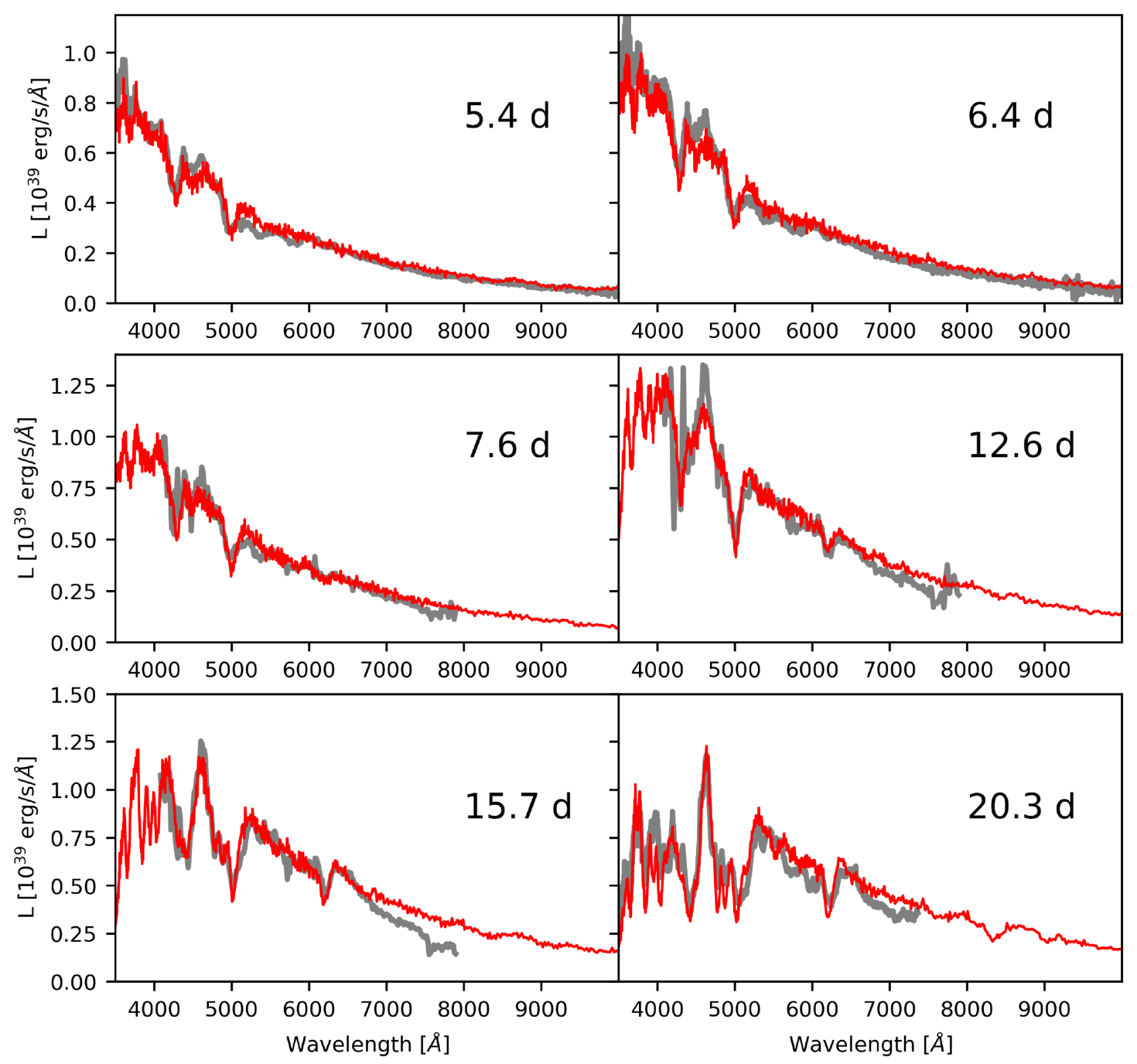

Figure 2. As Fig. 1, but for SN $2012 \mathrm{Z}$ obtained between -9 and $+6 \mathrm{~d}$ with respect to $B$-maximum.

0.01 and 0.10 , but silicon is always more abundant than sulphur. Magnesium appears with mass fractions of $0.05-0.10$ with higher uncertainty, but it also disappears at the highest velocities, just like other IMEs. Calcium abundances, which are also well constrained based on the fitting of the CaII H\&K lines and the Ca II NIR triplet, stay below 0.01 in all cases.

Note that, despite our use of a stratified abundance structure, our template estimated from the best-fit models does not differ strongly in the inner regions from the prediction of the pure deflagration models. Indeed, the amounts of most elements $(\mathrm{O}, \mathrm{Si}$, $\mathrm{S}, \mathrm{Ca}, \mathrm{Fe},{ }^{56} \mathrm{Ni}$ ), under a certain velocity, show mass fractions similar to those of the corresponding pure deflagration models of Fink et al. (2014).

However, a few discrepancies also appear in these comparisons. In the case of magnesium, a mass fraction one order of magnitude higher is necessary for our TARDIs model to fit the absorption feature of $\mathrm{Mg}$ II at $9500 \AA$ at least partially. Chromium and titanium show only a modest fraction in our best-fit models, although even these values exceed the predictions of the deflagration model by one and three orders of magnitude, respectively. The amount and the location of chromium are well constrained in our analysis, owing to several spectral features of $\mathrm{CrII}(\lambda 4588, \lambda 5237$ and the flux suppression at 3800 ̊).
Titanium is only required to fit the blue wing of the absorption feature at $\sim 4300 \AA$. The Ti II features in this wavelength region are strongly temperature-dependent (Hatano et al. 1999). Although the radiation temperatures derived from the TARDIS simulations are robust (see Section 3), the assumptions of the level populations, for which radiation temperature is the dominating parameter, are not (for further details see Kerzendorf \& Sim 2014). Thus, we cannot strongly constrain the amount of titanium based only on this feature.

Carbon is not allowed in the inner regions in our template model. This finding is a further discrepancy in the hydrodynamical calculations of the deflagration scenario, because carbon is the third most abundant element at any velocity in those models. Significant amounts of carbon cause strong characteristic spectral lines of CII $\lambda 4764, \lambda 6578$, as can be seen in Figs $11-14$.

\subsection{Outer regions of the abundance template}

Fig. 8 shows that the outer regions of the template model are dominated by oxygen, which reaches mass fractions of 0.80 . However, because we use oxygen as a 'filler' element (see Section 3.1), this feature might be artificial. Thus, we cannot draw any conclusion about the overabundance of oxygen. 

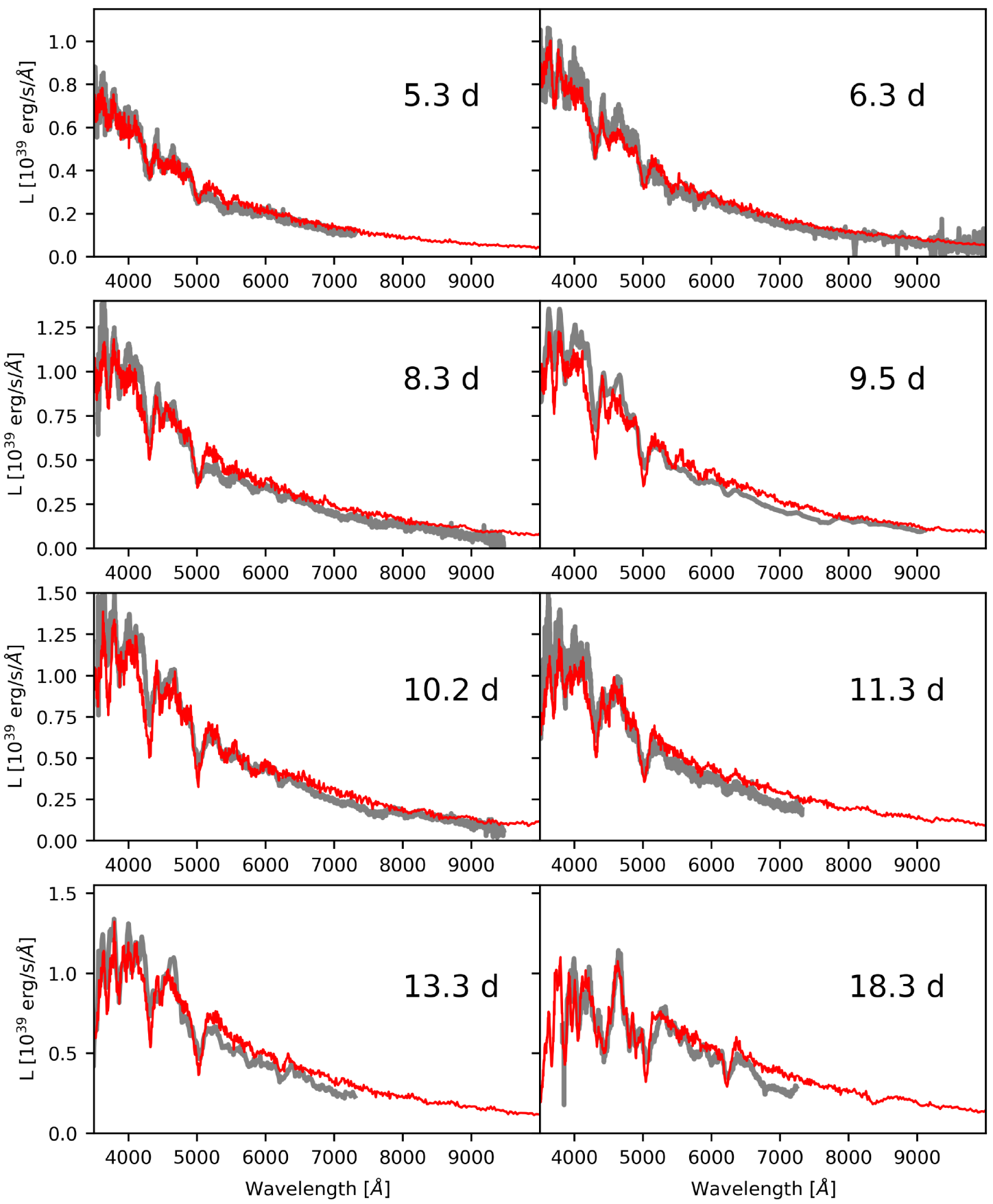

Figure 3. As Fig. 1, but for SN $2005 \mathrm{hk}$ obtained between -9 and $+4 \mathrm{~d}$ with respect to $B$-maximum.

Apart from oxygen, carbon and sodium are the only elements whose appearance is allowed in the outermost layers of the model ejecta. We increased the mass fraction of $\mathrm{Na}$ up to 0.05 in the colder outer layers in order to reproduce the characteristic NaI feature $\lambda 5890$ at later epochs. Note that this amount of $\mathrm{Na}$ is extreme compared with the deflagration models, which show values that are lower by two orders of magnitude.

Although carbon does not appear in the inner regions of the template model, its presence is allowed in the outer layers with nearly the same mass fraction as in the deflagration models. However, the complete elimination of carbon changes the goodness of the fits only slightly. To highlight the constraints on carbon in our best-fit models, we also plot spectra estimated from models with a constant carbon fraction, predicted by the deflagration models, and from models with zero carbon, in Figs 11-15.

\subsection{SN 2011ay}

Barna et al. (2017) fixed the density function of the model of SN 2011ay to the exponential fit of the W7 density profile (Nomoto, Thielemann \& Yokoi 1984). Because we are trying to connect our TARDIS models to hydrodynamical calculations, the densities are fitting parameters within the framework of the above-described deflagration-like profiles. As a result, the 

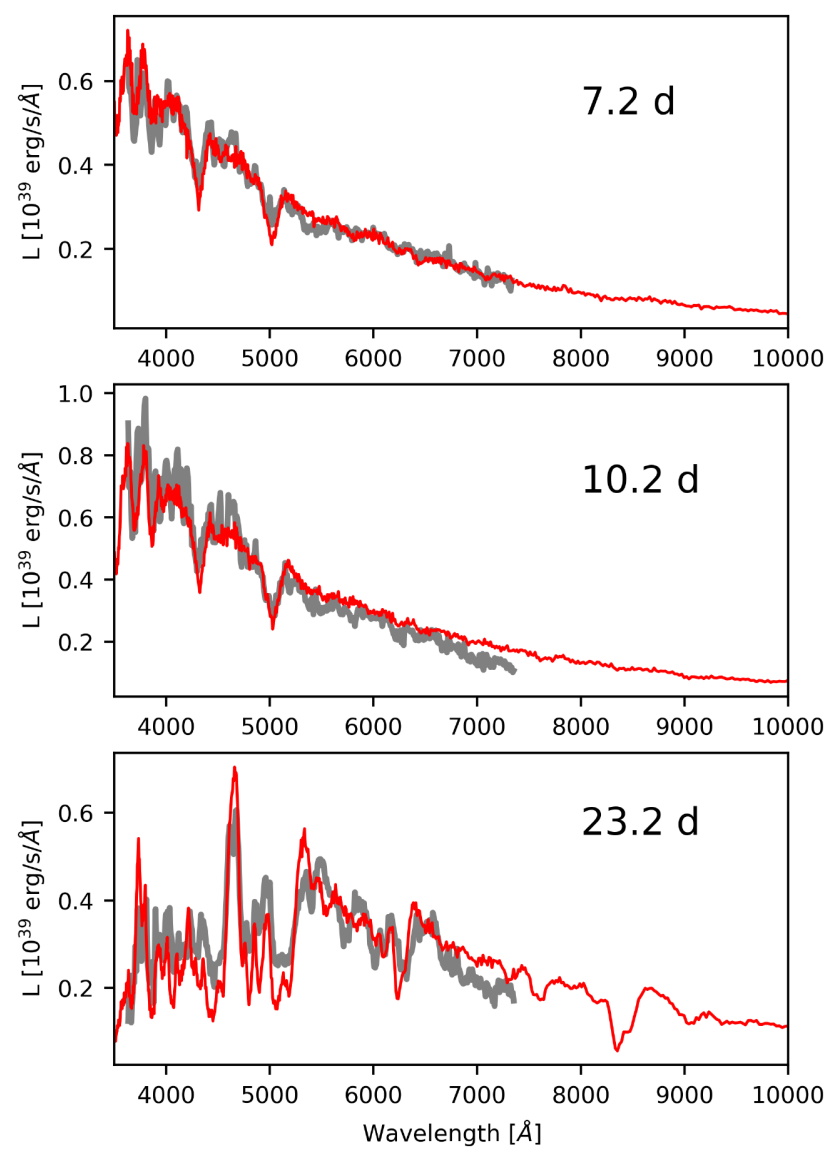

Figure 4. As Fig. 1, but for SN 2002cx obtained between -4 and $+12 d$ with respect to $B$-maximum.

new density function of the SN 2011ay model is similar to that of the N10def deflagration model (see Table 3), whose peak luminosity $\left(M_{\mathrm{V}}=-18.38 \mathrm{mag}\right)$ is the closest to that of $\mathrm{SN}$ 2011ay.

The abundance profiles are also subject to revision. However, despite the modification of the ejecta profile and the appearance of new elements (sodium and carbon) in our model, the primary attributes of the chemical abundances remain the same, and the conclusions stated in Barna et al. (2017) still hold. The adopted time of the explosion from the recent fitting process is $T_{0}=55632.7$ MJD, which is $0.2 \mathrm{~d}$ later than in our previous paper. The changes of the density profile affect mainly the longer wavelengths, at premaximum epochs, where the flux level of the synthetic spectra becomes closer to the observations (see Fig. 1). This is a significant improvement in the quality of the fits. However, around and after maximum light, the blue wings of the strongest absorption lines are less well fit, because of the lack of material at higher velocities. On the whole, the quality of the fits does not change significantly.

The abundance profile from our best-fit TARDIS model can be seen in Fig. 7. Comparing these mass fractions with those calculated from the N10def pure deflagration model, we find that the amounts of most elements $\left(\mathrm{O}, \mathrm{Si}, \mathrm{S}, \mathrm{Ca}, \mathrm{Fe},{ }^{56} \mathrm{Ni}\right)$ show mass fractions similar to in the deflagration model. The total mass of the radioactive nickel predicted by the $\mathrm{N} 10 \mathrm{def}$ model $\left(0.26 \mathrm{M}_{\odot}\right)$ is also close to the estimated value from the quasi-bolometric light curve $\left(0.22 \pm 0.01 \mathrm{M}_{\odot}\right.$; Szalai et al. 2015).

The abundances start to differ strongly only above the transition velocity, $\sim 11000 \mathrm{~km} \mathrm{~s}^{-1}$, where the ${ }^{56} \mathrm{Ni}$ abundance drops below
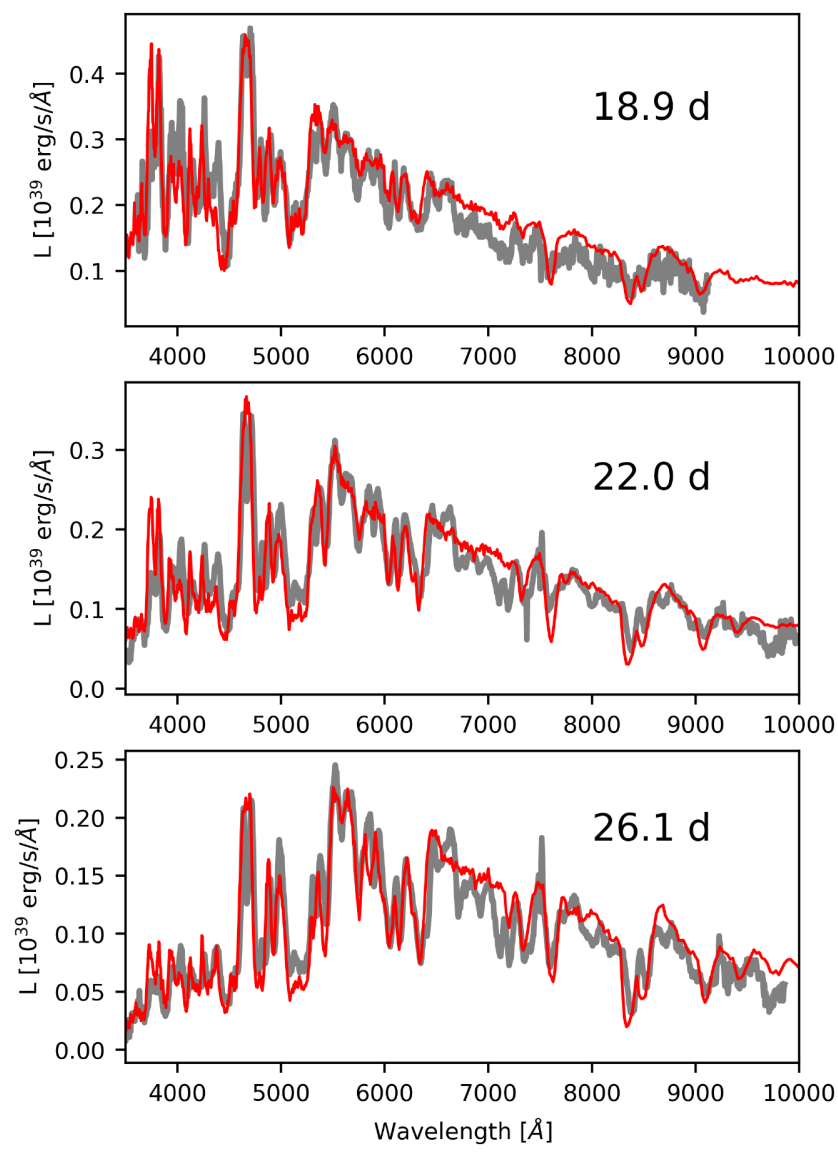

Figure 5. As Fig. 1, but for $\mathrm{SN} 2015 \mathrm{H}$ obtained between +3 and $+10 \mathrm{~d}$ with respect to $r$-maximum.

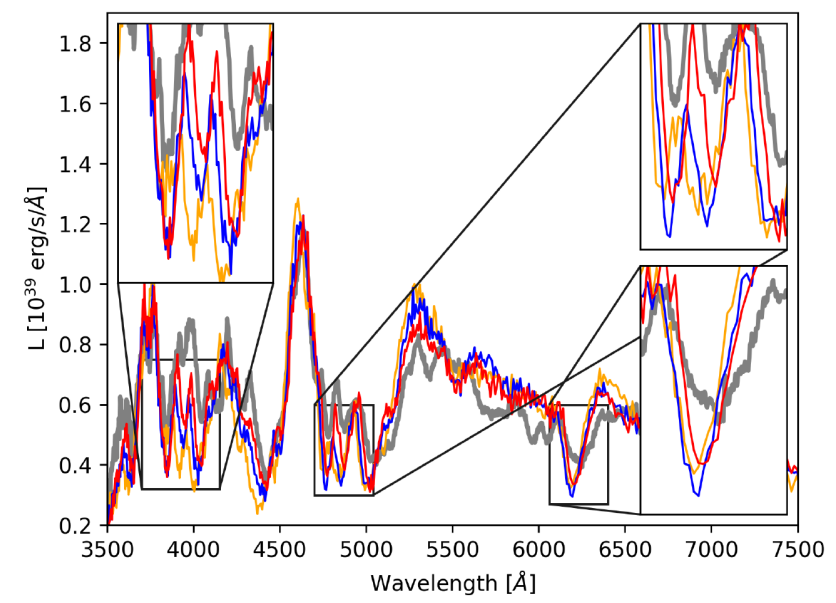

Figure 6. The spectrum of SN $2012 \mathrm{Z}$ at the epoch $+1.1 \mathrm{~d}$ with respect to $B$-maximum (grey), the synthetic spectrum from the best-fit model (red) and from the same model with the density profile of the N5def model (blue), and with the density profile without any cut-off at higher velocities (orange).

the increasing mass fraction of oxygen. A further discrepancy between our findings and the N10def model is the appearance of carbon (see Fig. 11), which is strictly constrained to the outer part of the ejecta in our analysis. However, above $12000 \mathrm{~km} \mathrm{~s}^{-1}$, even the carbon mass fraction $(0.10-0.15)$ meets the prediction of the deflagration model. 

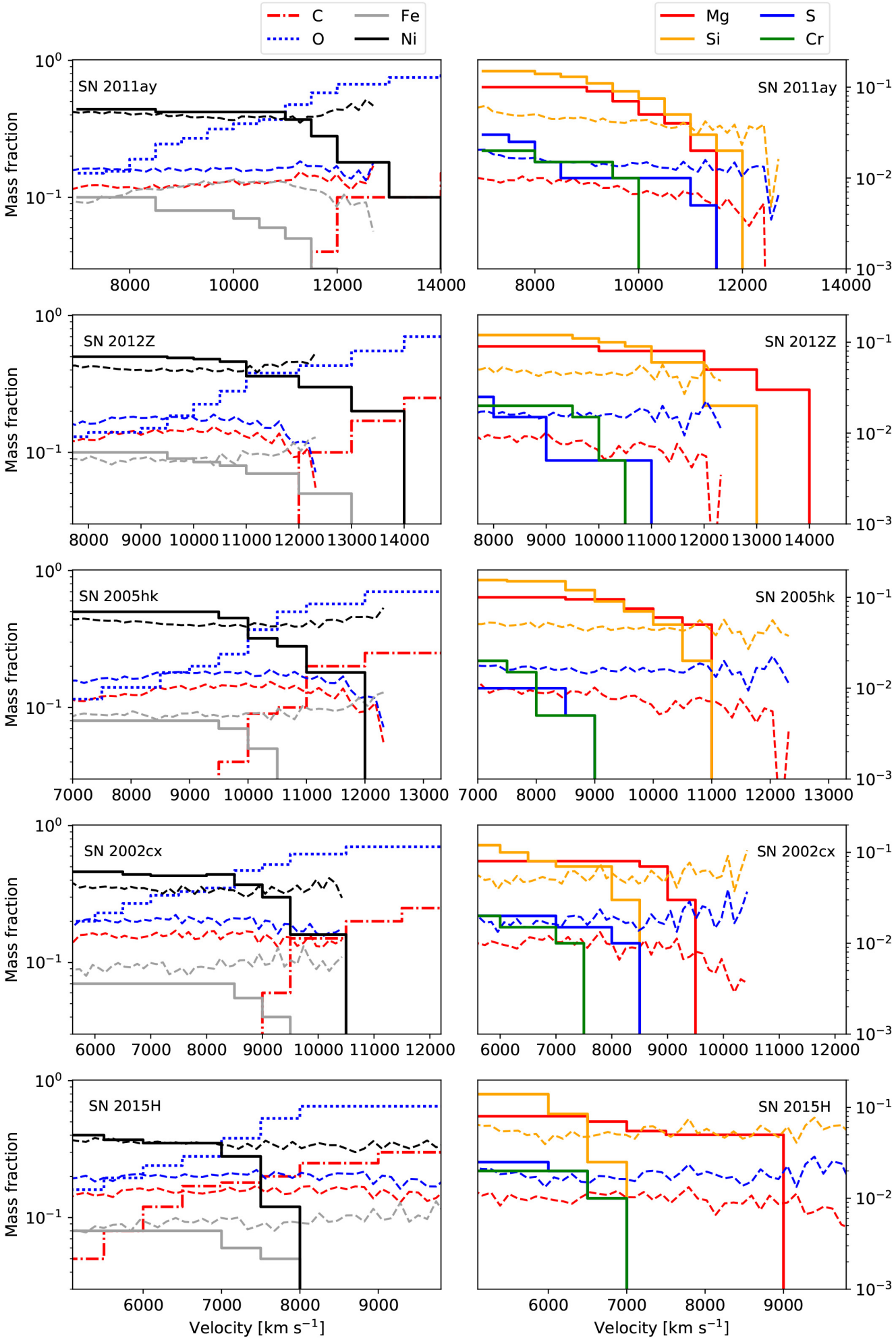

Figure 7. The stratified abundance profiles from the TARDIs fitting process of our SNe Iax sample (solid lines) compared with those originating from the corresponding (see Sections 4.4-4.8) pure deflagration model (dashed lines). Note that we use oxygen (dotted line) as a 'filler' element, while the carbon mass fraction (dash-dotted line) shows the limit for the prediction of the deflagration models. The $\mathrm{Fe} / \mathrm{Co} / \mathrm{Ni}$ mass fractions are computed at $100 \mathrm{~s}$ after the explosion.

\section{$4.5 \mathrm{SN} 2012 \mathrm{Z}$}

As can be seen in Fig. 2, the model fits for this supernova are not as good as for SN 2011ay in general. Although the primary features can be found in the synthetic spectra (see Fig. 12), several IGE lines are poorly fit before (especially iron) and after (cobalt and nickel) maximum. The continuum is well reproduced, but strong deviations appear at the near-infrared end of the spectrum near maximum light. 

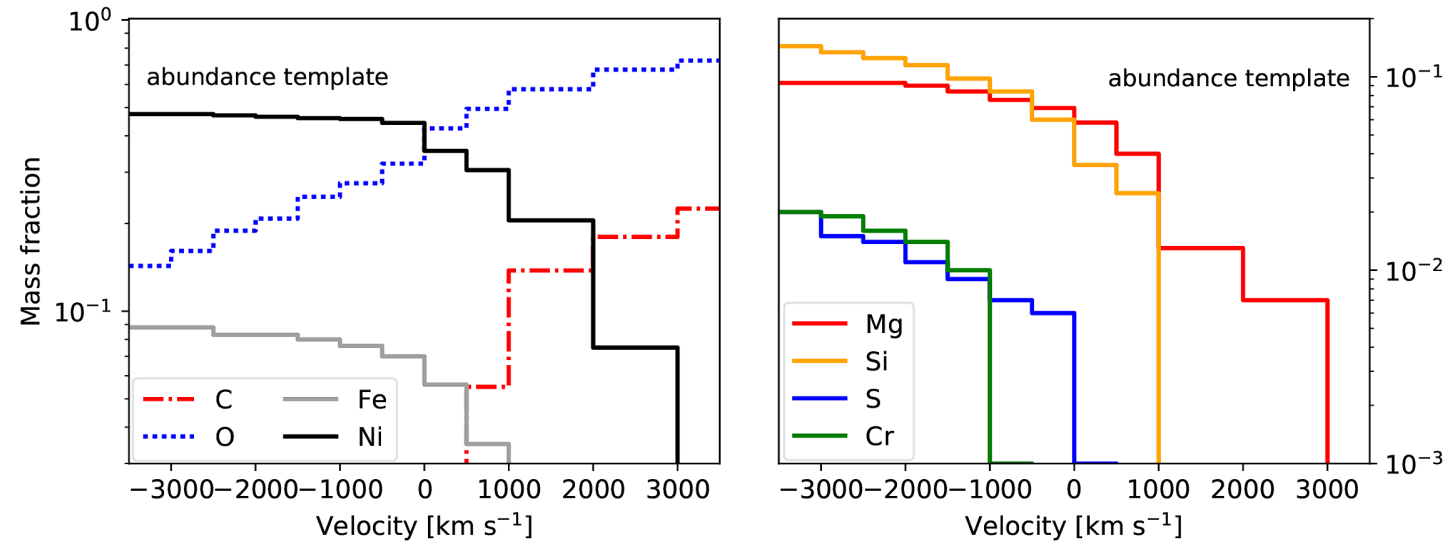

Figure 8. The calculated template abundance profile based on the average chemical abundances of the four most luminous SNe in our sample. The transition velocity, where $X(O)=X\left({ }^{56} \mathrm{Ni}\right)$, is chosen as the reference point, and thus it appears at $0 \mathrm{~km} \mathrm{~s}^{-1}$ in this figure. Note that we use oxygen as a 'filler' element, while the carbon mass fraction shows the limit for the prediction of the deflagration models. The $\mathrm{Fe} / \mathrm{Co} / \mathrm{Ni}$ mass fractions are computed at $100 \mathrm{~s}$ after the explosion.
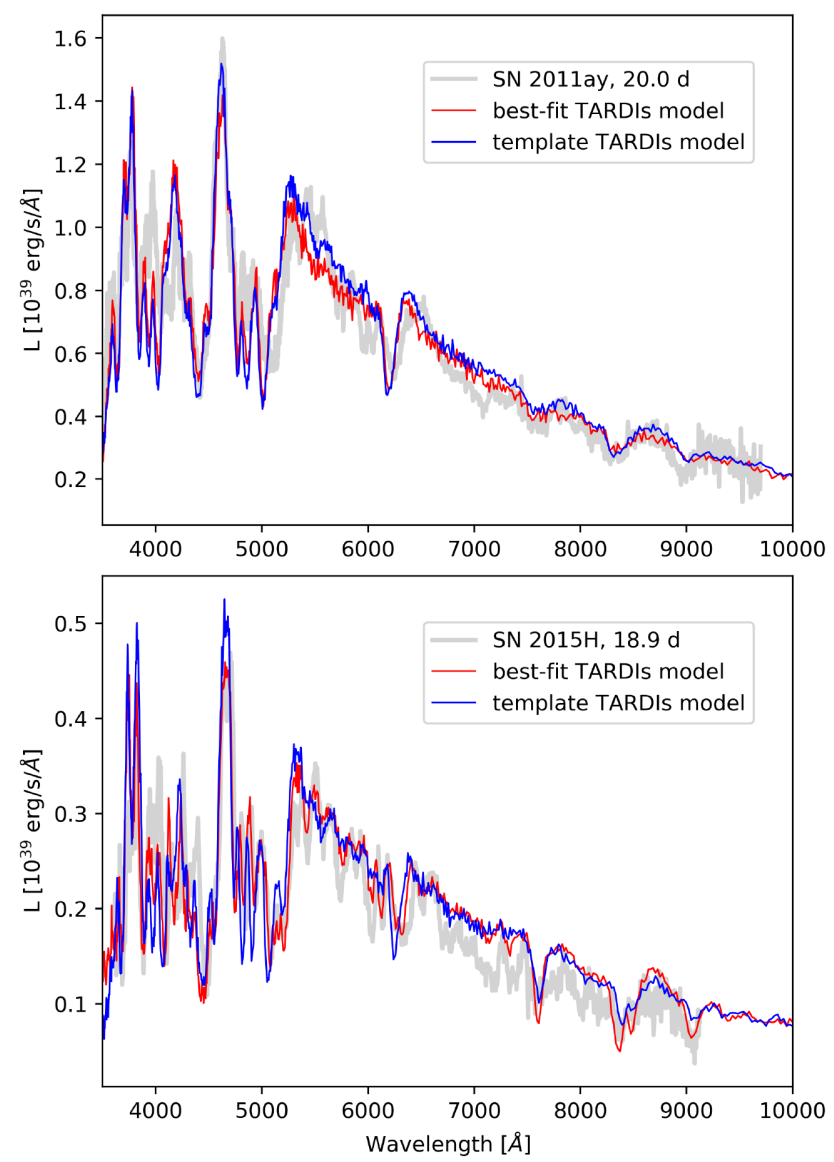

Figure 9. Spectra of SN 2011ay and SN 2015H obtained at nearly the same epoch (grey), compared to the synthetic spectra calculated with the best-fit (red) and the template abundance profiles (blue). The transition velocity of the template (Fig. 8) is shifted to 7000 and $11000 \mathrm{~km} \mathrm{~s}^{-1}$ for SN $2015 \mathrm{H}$ and SN 2011ay, respectively.

We found the date of the explosion as $T_{0}=55952.8$ MJD. Comparing the emergent luminosity parameter with that of SN 2011ay, SN $2012 Z$ seems slightly fainter at the same epochs. This contradicts the result of Stritzinger et al. $(2014,2015)$, but such
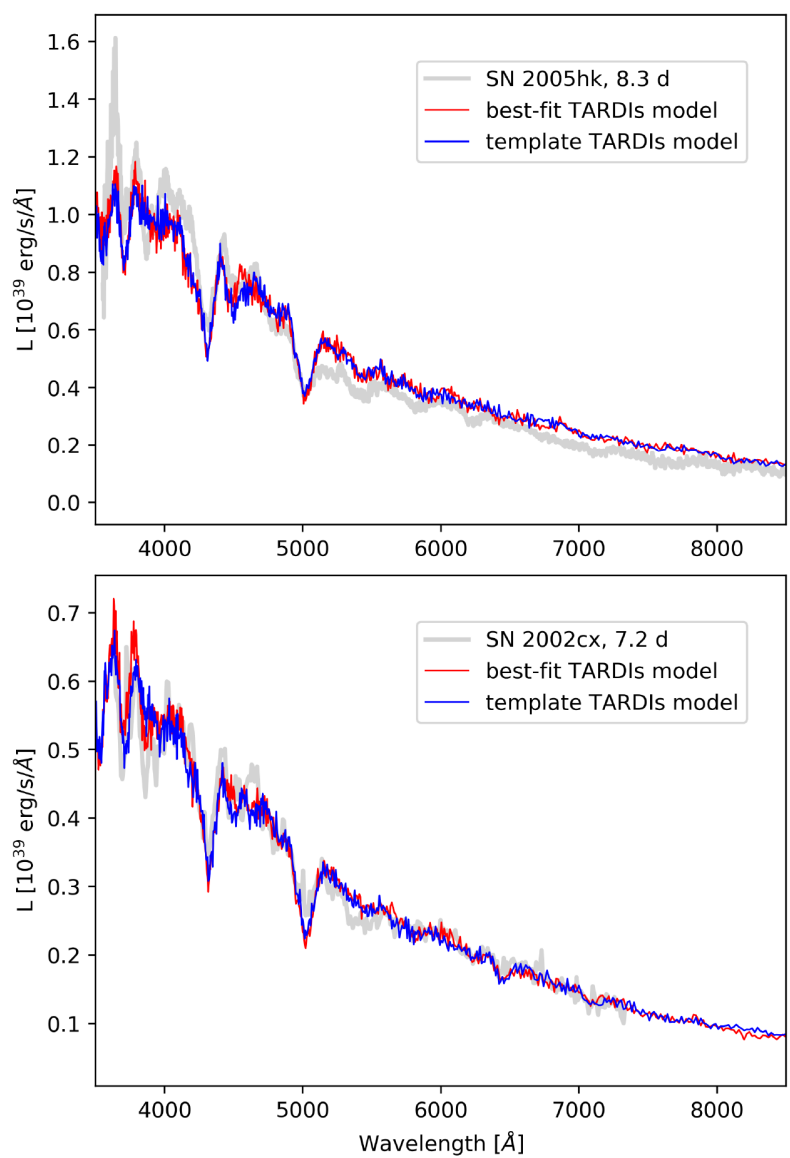

Figure 10. Spectra of SN 2005hk and SN 2002cx obtained at nearly the same epoch (grey), compared to the synthetic spectra calculated with the best-fit (red) and the template abundance profiles (blue). The transition velocity of the template (Fig. 8) is shifted to 8500 and $10000 \mathrm{~km} \mathrm{~s}^{-1}$ for SN 2002cx and SN 2005hk, respectively.

a moderate discrepancy could arise from the uncertainty in the distance.

The density profile of the model of SN $2012 \mathrm{Z}$ is between those of the N5def and N10def deflagration models, although with a steeper 


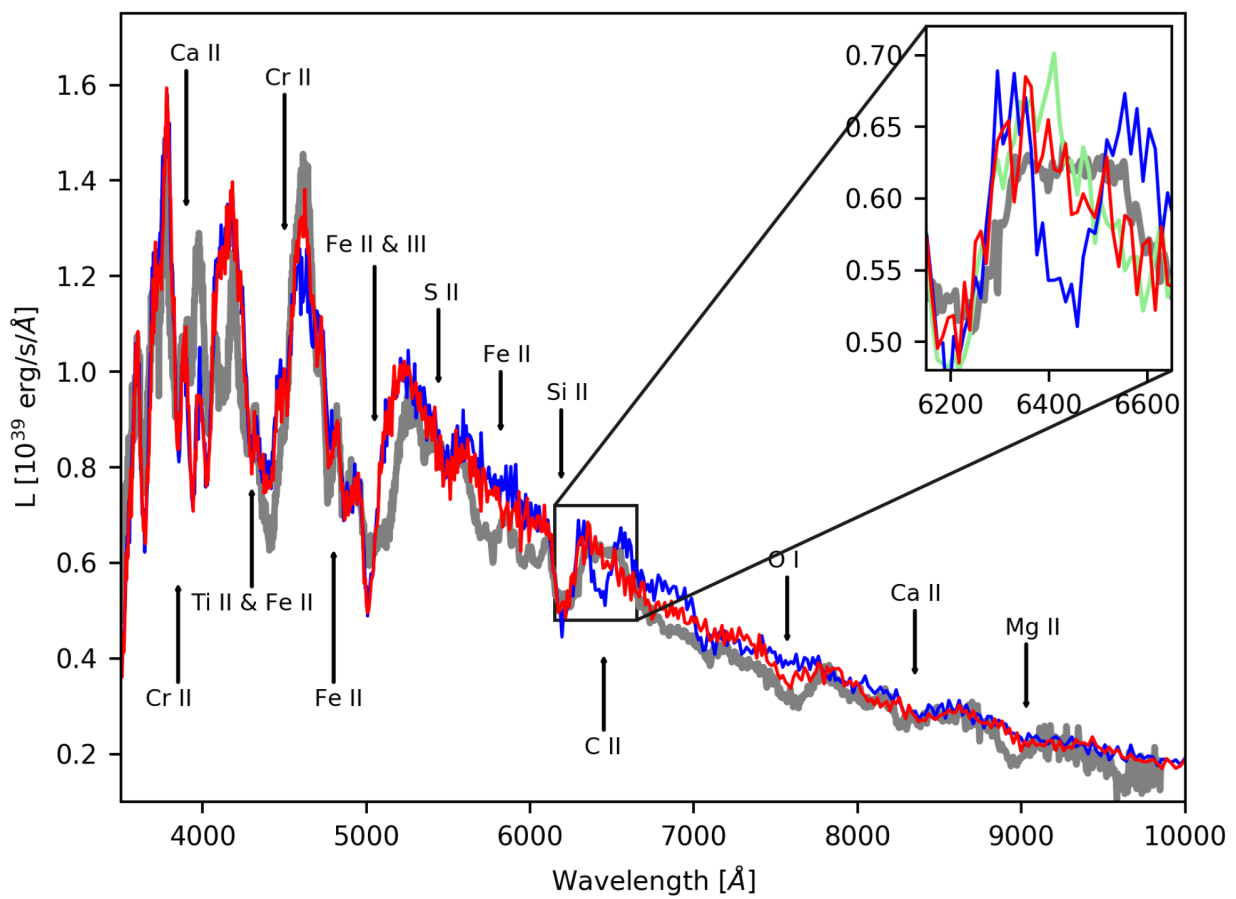

Figure 11. The spectrum of SN 2011 ay at the epoch $+2.6 \mathrm{~d}$ with respect to $B$-maximum (grey), the synthetic spectrum from the best-fit model (red), the same model with a constant carbon mass fraction of $\mathrm{X}(\mathrm{C})=0.12$ (blue) and with a zero carbon mass fraction (green, only in the cut-out). The black arrows show the positions of prominent absorption features and the related ions.

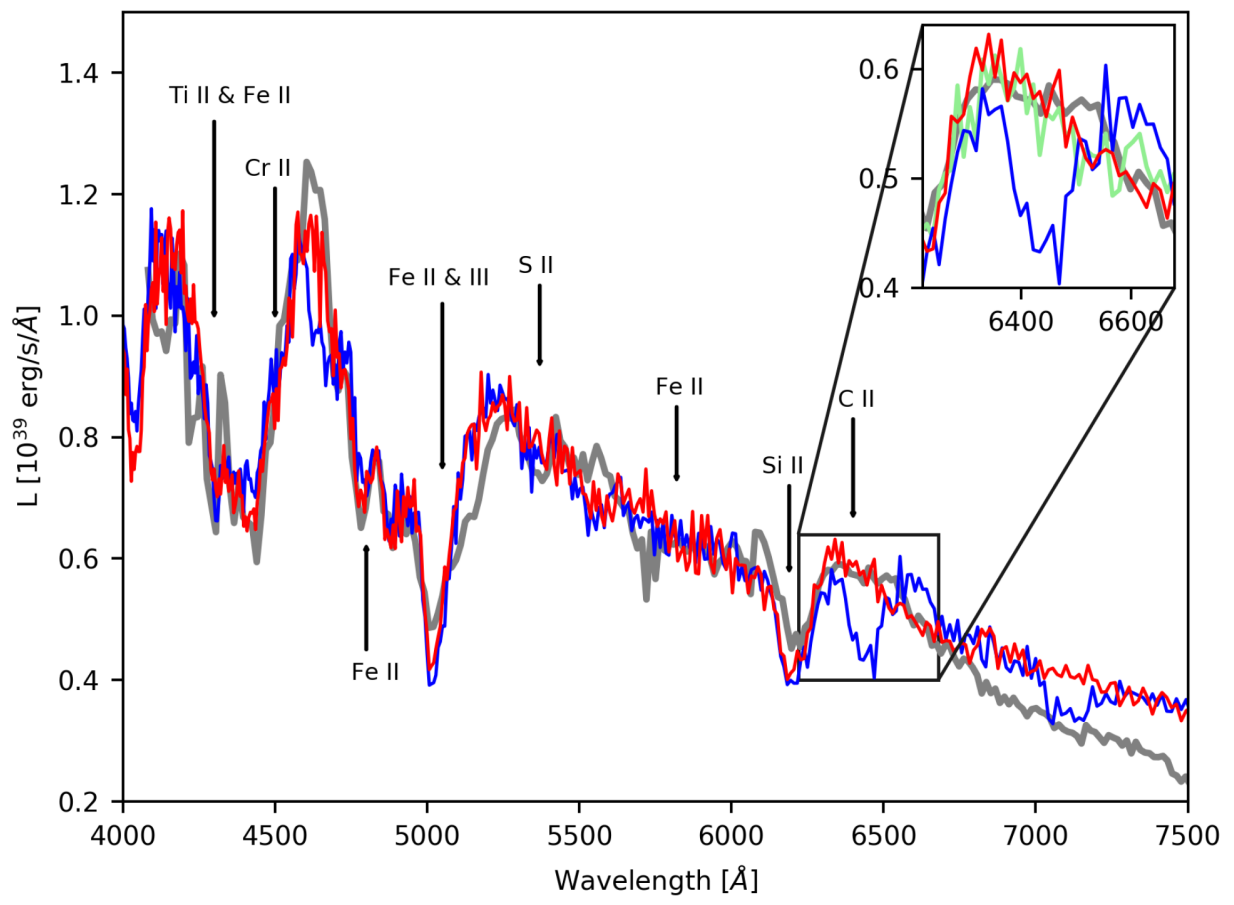

Figure 12. As Fig. 11, but for $\mathrm{SN} 2012 \mathrm{Z}$ at the epoch $+1.1 \mathrm{~d}$ with respect to $B$-maximum.

cut-off at higher velocities (see Table 3). This feature of the density profile is necessary to reproduce the blue wings of the spectral lines (Fig. 6). Because the photometry of SN $2012 \mathrm{Z}$ is better matched by the N10def model (peak absolute magnitude of $M_{\mathrm{V}}=-18.38$ $\mathrm{mag}$ ), the abundance profile is compared with its predictions in
Fig. 7. As for SN 2011ay, we find that the inner part of the ejecta has similarities to the predictions of the deflagration model. The transition velocity between the ${ }^{56} \mathrm{Ni}$-dominated inner and the $\mathrm{O}$ dominated outer part of the model ejecta is $11000 \mathrm{~km} \mathrm{~s}^{-1}$. Carbon appears only above this velocity limit in our model. 


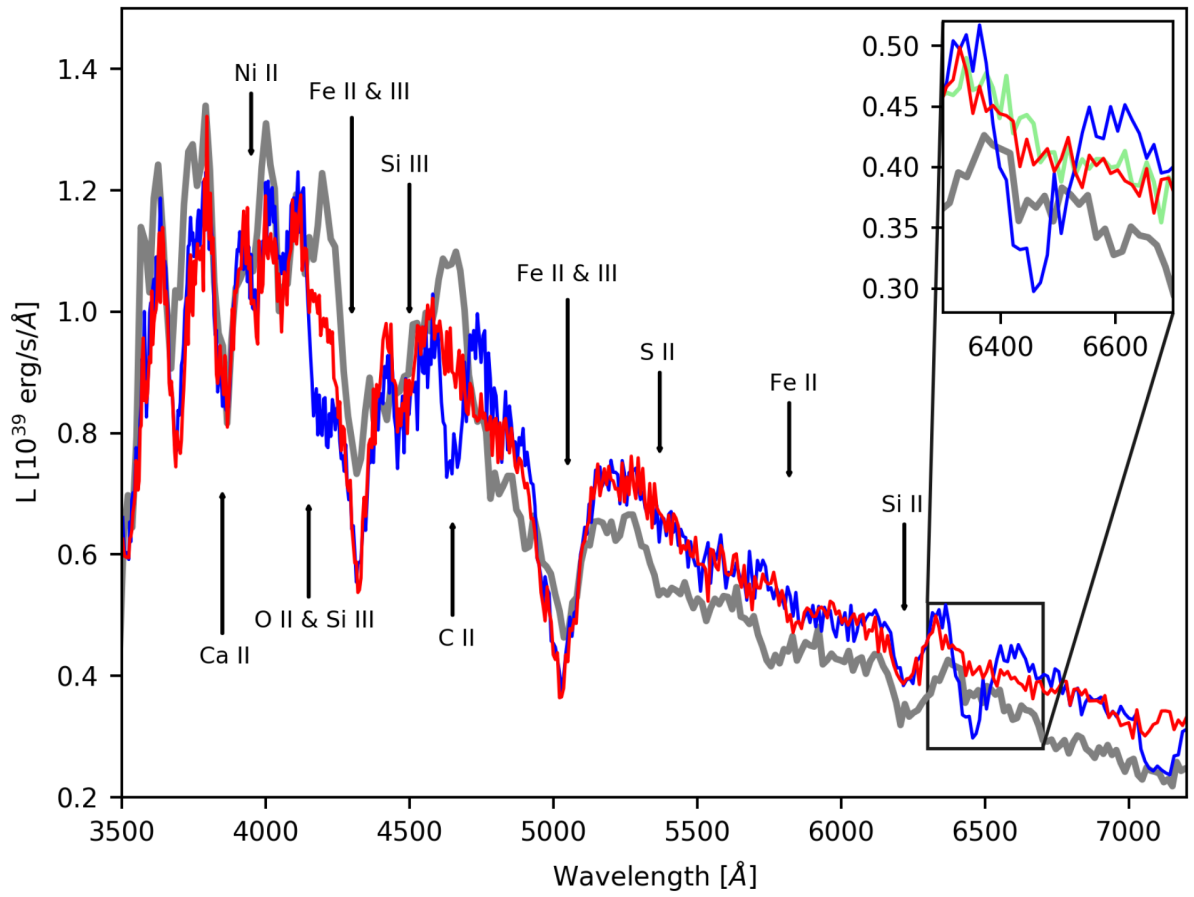

Figure 13. As Fig. 11, but for SN $2005 \mathrm{hk}$ at the epoch $-1.3 \mathrm{~d}$ with respect to $B$-maximum.

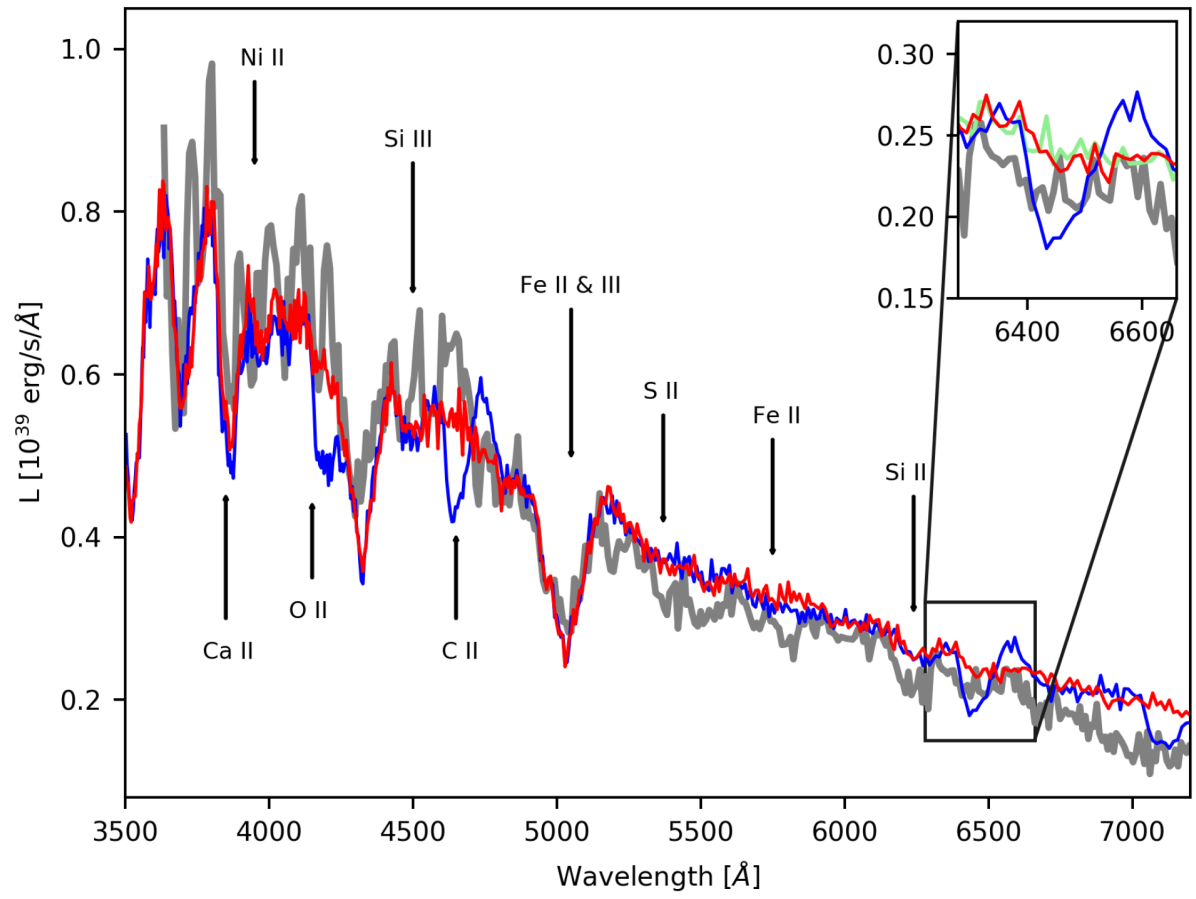

Figure 14. As Fig. 11, but for SN $2002 \mathrm{cx}$ at the epoch $-1.0 \mathrm{~d}$ with respect to $B$-maximum.

\subsection{SN 2005hk}

In the case of SN 2005hk, which is the second-best observed SN in our sample, the deviation between the observed spectra and our best-fit models is significant (see Fig. 3). This discrepancy occurs especially a few days before maximum light, when the continuum of our synthetic spectrum above $5500 \AA$ is consistently too high. The strongest IGE lines at shorter wavelengths are also not fitted well. Fixing these mismatches would require an extremely steep density profile, which would differ significantly from the adopted deflagration-like ejecta profiles, and the changes would spoil the relatively good fit at the earliest and latest epochs (see Fig. 13).

In Fig. 7, we show the abundance profile of our fits with the N5def model, whose peak absolute magnitudes $\left(M_{\mathrm{B}}=-17.85 \mathrm{mag}\right.$ and $M_{\mathrm{V}}=-18.24 \mathrm{mag}$ ) show the best agreement with SN 2005hk 


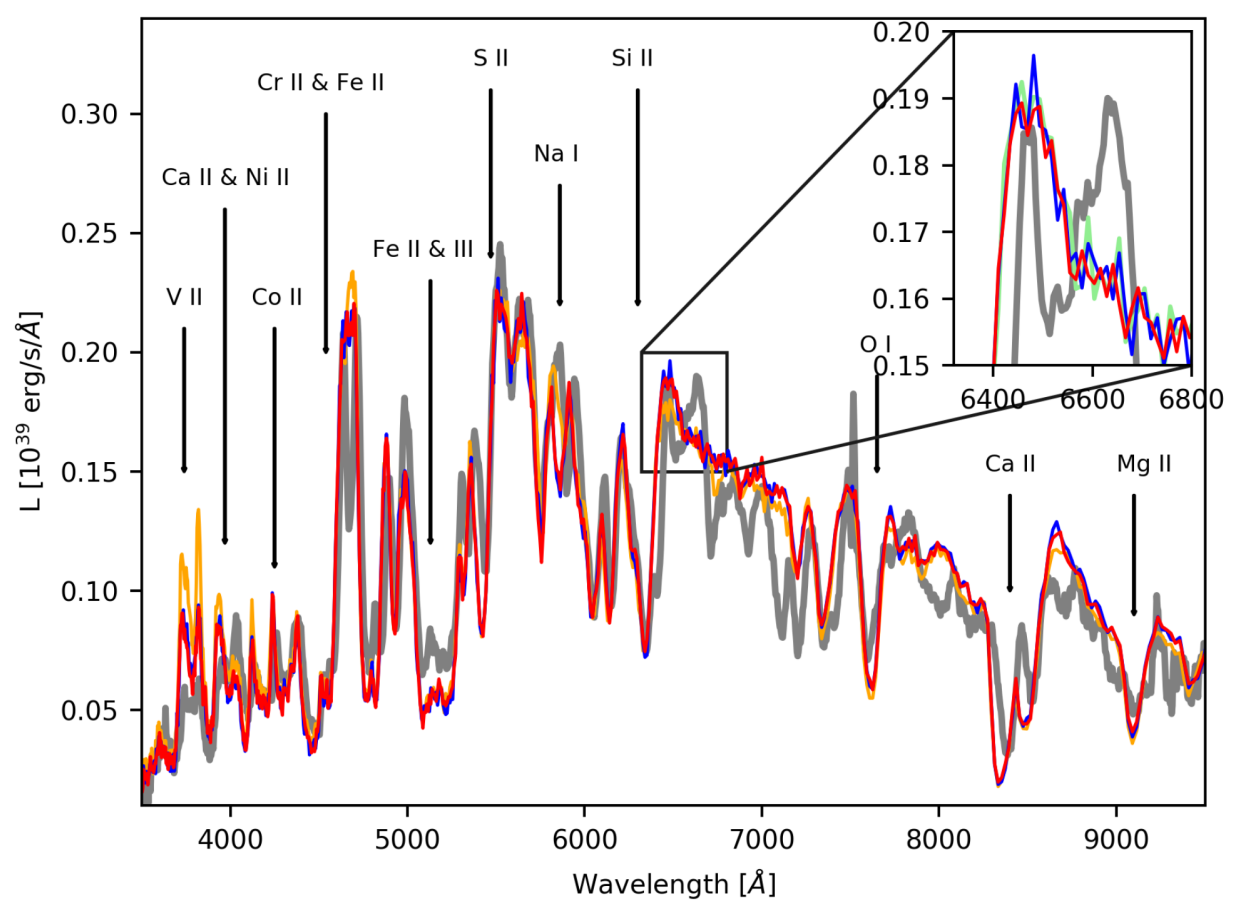

Figure 15. As Fig. 11, but for $\mathrm{SN} 2015 \mathrm{H}$ at the epoch $+10.4 \mathrm{~d}$ with respect to $r$-maximum. The vanadium-free TARDIs model is also plotted (orange).

$\left(M_{\mathrm{B}}=-18.02 \mathrm{mag}\right.$ and $\left.M_{\mathrm{V}}=-18.08 \mathrm{mag}\right)$. The transition velocity appears at $10000 \mathrm{~km} \mathrm{~s}^{-1}$, which is lower than for the more luminous SNe 2011 ay and 2012Z.

Magee et al. (2017) used TARDIS to compare the abundance profile from the N5def model with the spectrum obtained at $+3.6 \mathrm{~d}$ after $B$-maximum. The authors used $T_{0}=53670.0 \mathrm{MJD}$ as the time of explosion, which agrees with our value. They found that a deflagration model with a relatively restricted modelling volume between 7800 and $9400 \mathrm{~km} \mathrm{~s}^{-1}$ can broadly fit the flux continuum and the main features of IGEs and IMEs. However, they also showed that reducing the carbon mass fraction by an order of magnitude over the whole model volume improves the match of the $C_{\text {II }} \lambda 4750$ and $\lambda 6580$ features. This result is consistent with our best-fit model for SN 2005hk, in which the abundances in the region between 7800 and $9400 \mathrm{~km} \mathrm{~s}^{-1}$ are very similar to those of N5def, except for the case of carbon, which is nearly absent from this part of the ejecta in our model.

Apart from SN 2011ay, SN 2005hk is the only other SN Iax that has been the subject of a previously published abundance tomography analysis (Sahu et al. 2008). Thus, SN 2005hk offers a unique opportunity to compare our TARDIS model with the results of an independent investigation. However, it must be borne in mind that Sahu et al. (2008) used different radiation-matter approximations, which may cause systematic differences in the modelling process.

Sahu et al. (2008) adopted a much steeper density profile for their model ejecta, resulting in significantly more mass at lower velocities. Their densities are near or above the W7 model at the studied epochs, and are significantly higher than the values used here. Lower values of $v_{\text {phot }}$ were chosen in their fitting process than in our modelling. Despite the different modelling approach and physical parameters, the abundances show a surprisingly good agreement with our results. The earliest model atmosphere, which maps the highest-velocity regions, shows an extreme oxygen mass fraction of 0.86. Oxygen remains dominant in the later epochs after maximum light, while the amount of IGEs increases with time. The mass fractions of IMEs are the same order of magnitude as in our TARDIS model, while the mass fraction of carbon is under 0.01. All these similarities support the results of our modelling.

\subsection{SN 2002cx}

Only three spectra are available for SN 2002cx within the time range applicable for TARDIS analysis. Two of these were obtained before the $B$-band maximum, while the last was taken $12 \mathrm{~d}$ after maximum. This observational gap results in a jump of $\sim 2000 \mathrm{~km}$ $\mathrm{s}^{-1}$ between the $v_{\text {phot }}$ values of the corresponding epochs, causing significant uncertainty in our modelling process. All spectra have low signal-to-noise ratios, making the accurate fitting of optically thin lines more difficult. Despite these complicating factors, the quality of the fit is high regarding both the continuum level and the line profiles (see Fig. 14).

The abundance profile of our model can be seen in Fig. 7. The time of explosion is $T_{0}=52404.0 \mathrm{MJD}$. The adopted density profile for SN 2002cx is between those of N3def and N5def, but the absolute peak magnitude is closer to that of the less luminous deflagration model N3def $\left(M_{\mathrm{V}}=-17.52 \mathrm{mag}\right)$.

The transition velocity mentioned above, where our models start to deviate from the predictions of hydrodynamic calculations, appears at $8500 \mathrm{~km} \mathrm{~s}^{-1}$, namely $1500 \mathrm{~km} \mathrm{~s}^{-1}$ lower than for SN 2005hk. At the same time, the fitting of the $\mathrm{C}_{\text {II }}$ line at $6500 \AA$ gives strong constraints on the location of carbon above $9000 \mathrm{~km}$ $\mathrm{s}^{-1}$. Vanadium is used as an additional element to improve the fitting of the latest spectrum at $\sim 3800 \AA$, where VII suppresses the flux. Note that this is the only strong feature that supports the detection of V. Despite its small derived mass fraction (0.01-0.02), the V abundance in the inner part of the ejecta exceeds the prediction of the deflagration models by a few orders of magnitude. 


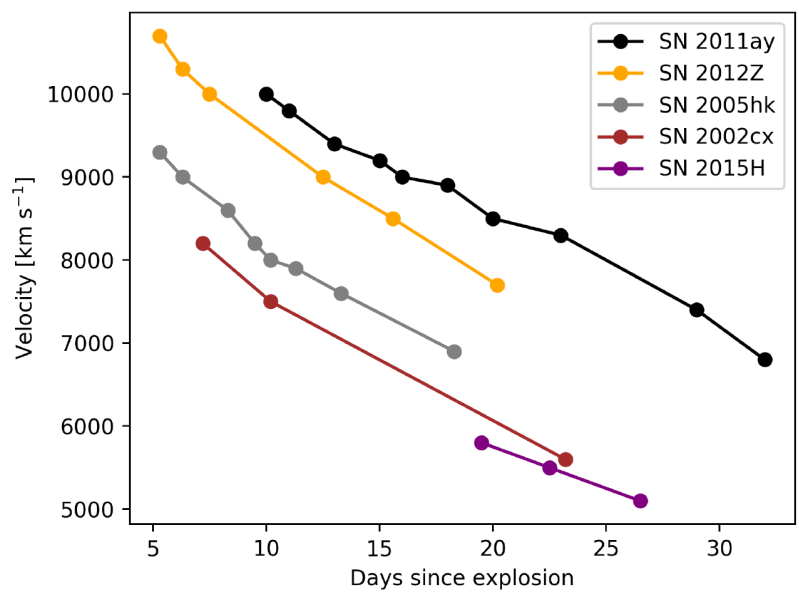

Figure 16. The photospheric velocities as a function of time since explosion from our TARDIS fits.

\subsection{SN 2015H}

The three available spectra for SN $2015 \mathrm{H}$ were obtained after maximum light and cover a relatively short time period. Thus, we lose crucial information about the outer regions and can rely only on the line profiles of IGEs and IMEs. Because of the higher uncertainties of the chemical profile, we omitted SN $2015 \mathrm{H}$ from the construction of the abundance template (see Section 4).

All our model spectra show good matches with the observations of SN 2015H (see Fig. 5); some absorption features are reproduced even around $\sim 7000 \AA$. Moreover, the three narrow spectral lines formed by $\mathrm{Na}$ I, Fe II and $\mathrm{Si}$ II also appear in our synthetic spectra at 5890, 5914 and $5979 \AA$ A, respectively (see Fig. 15).

Magee et al. (2016) fitted one spectrum of SN $2015 \mathrm{H}$ obtained at $+6 \mathrm{~d}$ with respect to $r$-maximum with TARDIS. They adopted $T_{0}=$ 57046.0 MJD as the explosion date, which is only $0.2 \mathrm{~d}$ earlier than our result. The authors used a constant abundance profile, which resulted in a nearly perfect fit using a velocity range of $2000 \mathrm{~km}$ $\mathrm{s}^{-1}$. Barna et al. (2017) showed that abundances in the outer ejecta can have a significant impact on the spectrum. In a broader range of $4000 \mathrm{~km} \mathrm{~s}^{-1}$, the stratified abundance profile seems a better solution. Compared with the photometrically close N3def deflagration model $\left(M_{\mathrm{V}}=-17.52 \mathrm{mag}\right)$, the similarity of the abundance profile is remarkable under the transition velocity of $7000 \mathrm{~km} \mathrm{~s}^{-1}$, as can be seen in Fig. 7.

Carbon can be allowed in almost the whole volume, with a decreasing fraction towards the centre, but, again, the uncertainties are higher in the case of SN 2015H. As for SN 2002cx, we use vanadium in the innermost regions of the modelled volume. The suspected features formed by VII ( $\lambda 3951$ and $\lambda 4883)$ appear in the spectra obtained at all three epochs.

\subsection{Evolution of photospheric velocity}

In all the studied $\mathrm{SNe}$, the $v_{\text {phot }}$ values decrease with time monotonically. The decline rates are higher at the earliest epochs: $\sim 200 \mathrm{~km}$ $\mathrm{s}^{-1} \mathrm{~d}^{-1}$ at $10 \mathrm{~d}$ before maximum light, decreasing to $\sim 100 \mathrm{~km} \mathrm{~s}^{-1}$ $\mathrm{d}^{-1}$ around maximum light, as best seen in Fig. 16 for SNe 2005hk and 2012Z. While different supernovae display different velocities, there are no significant differences in the decline rates of the various objects.

The $v_{\text {phot }}$ functions (Fig. 16) show a loose correlation with the peak luminosities in our sample (see Tables 1 and 3) at each epoch; fainter objects tend to have lower expansion velocities. This might support the idea that a correlation between peak luminosities and expansion velocities exists for the majority of SNe Iax. However, there are at least two examples reported in the literature, SN 2014ck (Tomasella et al. 2016) and SN 2009ku (Narayan et al. 2011), that show an extremely low photospheric velocity despite their having a relatively high peak luminosity. These examples imply that the physical origin of SNe Iax may be more complex, so that the whole subclass cannot be described with a few parameters.

The main parameter of the above-introduced template abundance profile is the transition velocity. This boundary between the inner regions dominated by IGEs and the outer areas dominated by oxygen appears at a different value in each object. The transition velocity also shows a loose correlation with the expansion velocities in our sample (see Section 4.4-4.8). These correlations point in the direction of SNe Iax being described with only a few parameters.

\section{CONCLUSIONS}

We have performed a comprehensive study of the chemical composition and the main physical properties of the diverse Type Iax $\mathrm{SN}$ subclass. We analysed the spectral time series of five SNe Iax with different peak luminosities and expansion velocities. The data were fitted by self-consistent atmosphere models calculated with the spectrum synthesis code TARDIS. We followed the modelling strategy introduced in Barna et al. (2017), applying a few modifications. We fitted the density of the SN ejecta within the framework of density profiles from hydrodynamic deflagration models, introducing new free parameters. However, our fits in general prefer a steeper cut-off in the density profiles at higher velocities than in the deflagration models.

The fitted synthetic spectra show a very good agreement with the observed spectral features. The continuum levels are mostly reproduced well; however, the relatively blue SN 2005hk shows a lower continuum flux beyond $\sim 5000 \AA$ than our models.

The TARDIS model atmospheres are built by varying the mass fractions of $\mathrm{C}, \mathrm{O}, \mathrm{Na}, \mathrm{Mg}, \mathrm{Si}, \mathrm{S}, \mathrm{Ca}, \mathrm{Ti}, \mathrm{Cr}, \mathrm{Fe}$ and ${ }^{56} \mathrm{Ni}$ for all five $\mathrm{SNe}$. The starting points of our fitting process are the predicted abundance profiles of the hydrodynamic calculations of pure deflagration models (Fink et al. 2014); we deviate from these values only if the change is indicated by the fitting of the spectral features. The best-fit models of the two least luminous SNe, 2002ck and $2015 \mathrm{H}$, also include vanadium with mass fractions of 0.01-0.02.

The most abundant element in our best-fit models is oxygen, just like in the previous abundance tomography for the spectral series of SN 2011ay (Barna et al. 2017). Here we fit the density profiles with a cut-off at higher velocities (Table 3 ) instead of using purely exponential profiles. We still have to use oxygen as a 'filler' element (Barna et al. 2017), despite the reduced mass in the outermost regions of the ejecta.

Broadly, a similar structure can be recognized in the abundance profiles of the five studied SNe. The trends of the abundance functions are monotonic for each element, and no abundance clump is found in the observed sample. The mass fractions of both IMEs and IGEs decrease towards higher velocities, with only oxygen and carbon showing upward trends. We can place only upper limits on the mass fractions of carbon, which does not appear in the inner ejecta volume (except in the case of SN 2015H) studied by the spectral series, its presence is allowed at higher velocities. The IGEs are dominated by the products of the ${ }^{56} \mathrm{Ni}$ decay chain, whose initial mass fraction is typically $0.40-0.50$ in the inner regions, while the initial amount of Fe is always below 10 per cent. Silicon is one of 
the best-constrained elements in our analysis because of the sharp SiII and Si III lines. The mass fraction reaches $0.10-0.15$ at lower velocities and continuously decreases outwards. Magnesium, sulphur and calcium show similar trends; their peak values in the innermost regions are $\sim 0.10, \sim 0.02$ and $\sim 0.01$, respectively.

We compared our findings with the abundance profiles of deflagration models of similar peak luminosity. We found that our stratified abundance structures deviate from the explosion models significantly at higher velocities and that the uniform abundances of the deflagration models do not describe well the outermost part of the Type Iax SN ejecta.

The mass fractions of the most abundant IMEs and IGEs in our TARDIS models are in relatively good agreement with the hydrodynamic calculations in the inner regions of the studied ejecta. However, we can tolerate carbon only in the outermost layers in our spectral fitting, while the deflagration calculations show a constant carbon abundance throughout the ejecta as a result of turbulent mixing.

Given that the derived abundance profiles from our model sample show very similar structures, we constructed a template abundance profile. For this purpose, we averaged the abundances of the individual models in each radial shell with the same relative velocity from the transition velocities (where $\mathrm{X}\left({ }^{56} \mathrm{Ni}\right)=\mathrm{X}(\mathrm{O})$ ). We generated a new set of synthetic spectra, shifting the abundance template (Fig. 8) in the velocity space according to the different transition velocities (see Section 4.1) of the five individual SN models. Their fittings with the observed data are nearly nearly as good as for our original best-fit models. The result that the abundance profile of these objects can be effectively described by one parameter (transition velocity) leads to the conclusion that the origins of the members of the diverse Type Iax subclass may not differ sharply from each other. Moreover, the transition velocities seem to correlate with the peak luminosities and the expansion velocities in our sample. A weakcorrelation between the peak brightnesses and the expansion velocities has been reported in the literature, although some outliers have been observed. Note that, at this point, we cannot classify the whole group of SNe Iax as a one-parameter family, in contrast to the case for normal SNe Ia. However, we are able to describe the five members of our Type Iax sample with only three parameters: the transition velocity of the abundance profile, the central density, and the location of the cut-off of the density profile. This result points in the direction that these peculiar transient objects can be described with only a few parameters.

\section{ACKNOWLEDGEMENTS}

This work is part of the project Transient Astrophysical Objects GINOP-2-3-2-15-2016-00033 of the National Research, Development and Innovation Office (NK- FIH), Hungary, funded by the European Union. BB received support from the Campus Mundi Short Study Programme of the Tempus Public Foundation through grant no. CM-SMR/252212/2017 and from the New National Excellence Program under UNKP-17-3 grant IV-SZTE-2. TS received funding from the Hungarian NKFIH/OTKA PD-112325 grant. WEK and BL acknowledge the Excellence Cluster Universe, Technische Universität München, Boltzmannstrasse 2, D-85748 Garching, Germany, and WEK acknowledges support from an ESO Fellowship. MK acknowledges support from the Klaus Tschira Foundation. SAS acknowledges support from STFC via grant ST/P000312/1. This study has made use of the Weizmann interactive supernova data repository (http://wiserep.weizmann.ac.il) and the Open Supernova Catalog.

\section{REFERENCES}

Barna B., Szalai T., Kromer M., Kerzendorf W., Vinkó J., Silverman J. M., Marion G. H., Wheelere J. C., 2017, MNRAS, 471, 4865

Blanchard P., Filippenko A. V., Pogge R. W., Garnavich P., Pedani M., Cenko S. B., Li W., 2011, Cent. Bureau Elect. Teleg., 2678

Blondin S. et al., 2012, AJ, 143, 126

Branch D., Baron E., Thomas R. C., Kasen D., Li W., Filippenko A. V., 2004, PASP, 116, 903

Branch D. et al., 2006, PASP, 118, 560

Burket J., Li W., 2005, IAU Circ., 8625

Cenko S. B. et al., 2012, Cent. Bureau Elect. Teleg., 3014

Chornock R., Filippenko A. V., Branch D., Foley R. J., Jha S., Li W., 2006, PASP, 118, 722

Fink M. et al., 2014, MNRAS, 438, 1762

Fisher A., 2000, PhD thesis, Univ. Oklahoma

Fitzpatrick E. L., Massa D., 2007, ApJ, 663, 320

Foley R. J. et al., 2009, AJ, 138, 376

Foley R. J., Brown P. J., Rest A., Challis P. J., Kirshner R. P., Wood-Vasey W. M., 2010, ApJ, 708, 1748

Foley R. J. et al., 2013, ApJ, 767, 57

Ganeshalingam M., Li W., Filippenko A. V., 2011, MNRAS, 416, 2607

Guillochon J., Parrent J., Kelley L. Z., Margutti R., 2017, ApJ, 835, 64

Hatano K., Branch D., Fisher A., Millard J., Baron E., 1999, ApJS, 121, 233

Jha S., Branch D., Chornock R., Foley R. J., Li W., Swift B. J., Casebeer D., Filippenko A. V., 2006, AJ, 132, 189

Jordan IV G. C., Perets H. B., Fisher R. T., van Rossum D. R., 2012, ApJ, 761, L23

Kerzendorf W. E., Sim S. A., 2014, MNRAS, 440, 387

Kromer M. et al., 2013, MNRAS, 429, 2287

Kromer M. et al., 2015, MNRAS, 450, 3045

Li W. et al., 2003, PASP, 115, 453

Liu Z.-W., Moriya T. J., Stancliffe R. J., Wang B., 2015, A\&A, 574, A12

Long M., et al., 2014, ApJ, 789, 103

Magee M. R. et al., 2016, A\&A, 589, 89

Magee M. R. et al., 2017, A\&A, 601, 62

Mazzali P. A., 2000, A\&A, 363, 705

McClelland C. M. et al., 2010, ApJ, 720, 704

McCully C. et al., 2014, ApJ, 786, 134

McCully C. et al., 2014, Nature, 512, 54

Narayan G. et al., 2011, ApJ, 731, L11

Nomoto K., Thielemann F.-K., Yokoi K., 1984, ApJ, 286, 644

Parker S., 2015, Cent. Bureau Elect. Teleg., 4093, 2

Parrent J. T., 2011, ApJ, 732, 15

Phillips M. M. et al., 2007, PASP, 119, 360

Phillips M. M. et al., 2013, ApJ, 779, 38

Sahu D. K. et al., 2008, ApJ, 680, 580

Schlafly E. F., Finkbeiner D. P., 2011, ApJ, 737, 103

Silverman J. M. et al., 2012, MNRAS, 425, 1789

Stehle M., Mazzali P. A., Benetti S., Hillebrandt W., 2005, MNRAS, 360, 1231

Stritzinger M. D. et al., 2014, A\&A, 561, A146

Stritzinger M. D. et al., 2015, A\&A, 573, A2

Szalai T. et al., 2015, MNRAS, 453, 2103

Thomas R. C. et al., 2011, ApJ, 743, 27

Tomasella L. et al., 2016, MNRAS, 459, 1018

Valenti S. et al., 2009, Nature, 459, 674

Wang B., Justham S., Han Z., 2013, A\&A, 559, 94

Wood-Vasey W. M., Aldering G., Nugent P., Helin E.F., Pravdo S., Hicks M., Lawrence K., 2002, IAU Circ. 7902

Yamanaka M. et al., 2015, ApJ, 806, 191

Yaron O., Gal-Yam A., 2012, PASP, 124, 668

\section{APPENDIX A: ABUNDANCE PARAMETERS OF THE BEST-FIT MODELS}


Table A1. Mass fractions of chemical elements in velocity layers (between $\mathrm{v}_{\text {low }}$ and $\mathrm{v}_{\mathrm{up}}$ in $\mathrm{km} \mathrm{s}^{-1}$ ) of the best-fit model for SN 2011 ay.

\begin{tabular}{|c|c|c|c|c|c|c|c|c|c|c|c|c|}
\hline $\mathrm{X}(\mathrm{C})$ & $\mathrm{X}(\mathrm{O})$ & $\mathrm{X}(\mathrm{Na})$ & $\mathrm{X}(\mathrm{Mg})$ & $\mathrm{X}(\mathrm{Si})$ & $\mathrm{X}(\mathrm{S})$ & $\mathrm{X}(\mathrm{Ca})$ & $\mathrm{X}(\mathrm{Ti})$ & $\mathrm{X}(\mathrm{Cr})$ & $\mathrm{X}(\mathrm{Fe})$ & $X\left({ }^{56} \mathrm{Ni}\right)$ & $v_{\text {low }}$ & $\mathrm{v}_{\text {up }}$ \\
\hline 0.000 & 0.150 & 0.000 & 0.100 & 0.150 & 0.030 & 0.005 & 0.020 & 0.020 & 0.100 & 0.440 & 7000 & 7500 \\
\hline 0.000 & 0.155 & 0.000 & 0.100 & 0.150 & 0.025 & 0.005 & 0.020 & 0.020 & 0.100 & 0.440 & 7500 & 8000 \\
\hline 0.000 & 0.190 & 0.000 & 0.100 & 0.140 & 0.015 & 0.005 & 0.010 & 0.015 & 0.100 & 0.440 & 8000 & 8500 \\
\hline 0.000 & 0.245 & 0.000 & 0.100 & 0.130 & 0.010 & 0.003 & 0.010 & 0.015 & 0.080 & 0.420 & 8500 & 9000 \\
\hline 0.000 & 0.270 & 0.000 & 0.090 & 0.110 & 0.010 & 0.003 & 0.005 & 0.015 & 0.080 & 0.420 & 9000 & 9500 \\
\hline 0.000 & 0.315 & 0.000 & 0.070 & 0.090 & 0.010 & 0.003 & 0.000 & 0.010 & 0.080 & 0.420 & 9500 & 10000 \\
\hline 0.000 & 0.345 & 0.030 & 0.050 & 0.075 & 0.010 & 0.003 & 0.000 & 0.000 & 0.070 & 0.420 & 10000 & 10500 \\
\hline 0.000 & 0.370 & 0.050 & 0.040 & 0.050 & 0.010 & 0.002 & 0.000 & 0.000 & 0.060 & 0.420 & 10500 & 11000 \\
\hline 0.000 & 0.475 & 0.050 & 0.020 & 0.030 & 0.005 & 0.001 & 0.000 & 0.000 & 0.050 & 0.370 & 11000 & 11500 \\
\hline 0.040 & 0.580 & 0.050 & 0.000 & 0.020 & 0.000 & 0.000 & 0.000 & 0.000 & 0.030 & 0.280 & 11500 & 12000 \\
\hline 0.100 & 0.670 & 0.050 & 0.000 & 0.000 & 0.000 & 0.000 & 0.000 & 0.000 & 0.000 & 0.180 & 12000 & 13000 \\
\hline 0.100 & 0.750 & 0.050 & 0.000 & 0.000 & 0.000 & 0.000 & 0.000 & 0.000 & 0.000 & 0.100 & 13000 & 14000 \\
\hline 0.150 & 0.800 & 0.050 & 0.000 & 0.000 & 0.000 & 0.000 & 0.000 & 0.000 & 0.000 & 0.000 & 14000 & 15000 \\
\hline
\end{tabular}

Table A2. Mass fractions of chemical elements in velocity layers (between $v_{\text {low }}$ and $\mathrm{v}_{\text {up }}$ in $\mathrm{km} \mathrm{s}^{-1}$ ) of the best-fit model for SN $2012 \mathrm{Z}$.

\begin{tabular}{|c|c|c|c|c|c|c|c|c|c|c|c|c|}
\hline $\mathrm{X}(\mathrm{C})$ & $\mathrm{X}(\mathrm{O})$ & $\mathrm{X}(\mathrm{Na})$ & $\mathrm{X}(\mathrm{Mg})$ & $\mathrm{X}(\mathrm{Si})$ & $\mathrm{X}(\mathrm{S})$ & $\mathrm{X}(\mathrm{Ca})$ & $\mathrm{X}(\mathrm{Ti})$ & $\mathrm{X}(\mathrm{Cr})$ & $\mathrm{X}(\mathrm{Fe})$ & $\mathrm{X}\left({ }^{56} \mathrm{Ni}\right)$ & $\mathrm{v}_{\text {low }}$ & $\mathrm{v}_{\text {up }}$ \\
\hline 0.000 & 0.130 & 0.000 & 0.090 & 0.120 & 0.025 & 0.003 & 0.010 & 0.020 & 0.100 & 0.500 & 7500 & 8000 \\
\hline 0.000 & 0.140 & 0.000 & 0.090 & 0.120 & 0.015 & 0.003 & 0.010 & 0.020 & 0.100 & 0.500 & 8000 & 8500 \\
\hline 0.000 & 0.140 & 0.000 & 0.090 & 0.120 & 0.015 & 0.003 & 0.010 & 0.020 & 0.100 & 0.500 & 8500 & 9000 \\
\hline 0.000 & 0.150 & 0.000 & 0.090 & 0.120 & 0.005 & 0.003 & 0.010 & 0.020 & 0.100 & 0.500 & 9000 & 9500 \\
\hline 0.000 & 0.185 & 0.000 & 0.090 & 0.110 & 0.005 & 0.003 & 0.010 & 0.015 & 0.090 & 0.490 & 9500 & 10000 \\
\hline 0.000 & 0.225 & 0.000 & 0.080 & 0.100 & 0.005 & 0.003 & 0.005 & 0.005 & 0.085 & 0.480 & 10000 & 10500 \\
\hline 0.000 & 0.280 & 0.000 & 0.080 & 0.090 & 0.005 & 0.003 & 0.000 & 0.000 & 0.080 & 0.460 & 10500 & 11000 \\
\hline 0.020 & 0.380 & 0.030 & 0.080 & 0.060 & 0.000 & 0.002 & 0.000 & 0.000 & 0.070 & 0.360 & 11000 & 12000 \\
\hline 0.100 & 0.430 & 0.050 & 0.050 & 0.020 & 0.000 & 0.001 & 0.000 & 0.000 & 0.050 & 0.300 & 12000 & 13000 \\
\hline 0.170 & 0.550 & 0.050 & 0.030 & 0.000 & 0.000 & 0.000 & 0.000 & 0.000 & 0.000 & 0.200 & 13000 & 14000 \\
\hline 0.250 & 0.700 & 0.050 & 0.000 & 0.000 & 0.000 & 0.000 & 0.000 & 0.000 & 0.000 & 0.000 & 14000 & 15000 \\
\hline 0.250 & 0.700 & 0.050 & 0.000 & 0.000 & 0.000 & 0.000 & 0.000 & 0.000 & 0.000 & 0.000 & 15000 & 16000 \\
\hline
\end{tabular}

Table A3. Mass fractions of chemical elements in velocity layers (between $v_{\text {low }}$ and $\mathrm{v}_{\mathrm{up}}$ in $\mathrm{km} \mathrm{s}^{-1}$ ) of the best-fit model for SN $2005 \mathrm{hk}$.

\begin{tabular}{|c|c|c|c|c|c|c|c|c|c|c|c|c|}
\hline $\mathrm{X}(\mathrm{C})$ & $\mathrm{X}(\mathrm{O})$ & $\mathrm{X}(\mathrm{Na})$ & $\mathrm{X}(\mathrm{Mg})$ & $\mathrm{X}(\mathrm{Si})$ & $\mathrm{X}(\mathrm{S})$ & $\mathrm{X}(\mathrm{Ca})$ & $\mathrm{X}(\mathrm{Ti})$ & $\mathrm{X}(\mathrm{Cr})$ & $\mathrm{X}(\mathrm{Fe})$ & $\mathrm{X}\left({ }^{56} \mathrm{Ni}\right)$ & $\mathrm{v}_{\text {low }}$ & $\mathrm{v}_{\text {up }}$ \\
\hline 0.000 & 0.115 & 0.000 & 0.100 & 0.155 & 0.010 & 0.005 & 0.015 & 0.020 & 0.080 & 0.500 & 7000 & 7500 \\
\hline 0.000 & 0.140 & 0.000 & 0.100 & 0.150 & 0.010 & 0.005 & 0.015 & 0.015 & 0.080 & 0.500 & 7500 & 8000 \\
\hline 0.000 & 0.140 & 0.000 & 0.100 & 0.150 & 0.010 & 0.005 & 0.010 & 0.005 & 0.080 & 0.500 & 8000 & 8500 \\
\hline 0.000 & 0.180 & 0.000 & 0.095 & 0.120 & 0.005 & 0.005 & 0.010 & 0.005 & 0.080 & 0.500 & 8500 & 9000 \\
\hline 0.000 & 0.200 & 0.030 & 0.095 & 0.090 & 0.000 & 0.005 & 0.000 & 0.000 & 0.080 & 0.500 & 9000 & 9500 \\
\hline 0.040 & 0.245 & 0.050 & 0.075 & 0.070 & 0.000 & 0.003 & 0.000 & 0.000 & 0.070 & 0.450 & 9500 & 10000 \\
\hline 0.090 & 0.370 & 0.050 & 0.060 & 0.050 & 0.000 & 0.001 & 0.000 & 0.000 & 0.050 & 0.320 & 10000 & 10500 \\
\hline 0.100 & 0.500 & 0.050 & 0.050 & 0.020 & 0.000 & 0.000 & 0.000 & 0.000 & 0.000 & 0.280 & 10500 & 11000 \\
\hline 0.200 & 0.570 & 0.050 & 0.000 & 0.000 & 0.000 & 0.000 & 0.000 & 0.000 & 0.000 & 0.180 & 11000 & 12000 \\
\hline 0.250 & 0.700 & 0.050 & 0.000 & 0.000 & 0.000 & 0.000 & 0.000 & 0.000 & 0.000 & 0.000 & 12000 & 13000 \\
\hline 0.250 & 0.700 & 0.050 & 0.000 & 0.000 & 0.000 & 0.000 & 0.000 & 0.000 & 0.000 & 0.000 & 13000 & 14000 \\
\hline
\end{tabular}


Table A4. Mass fractions of chemical elements in velocity layers (between $\mathrm{v}_{\text {low }}$ and $\mathrm{v}_{\text {up }}$ in $\mathrm{km} \mathrm{s}^{-1}$ ) of the best-fit model for SN $2002 \mathrm{cx}$.

\begin{tabular}{|c|c|c|c|c|c|c|c|c|c|c|c|c|c|}
\hline $\mathrm{X}(\mathrm{C})$ & $\mathrm{X}(\mathrm{O})$ & $\mathrm{X}(\mathrm{Na})$ & $\mathrm{X}(\mathrm{Mg})$ & $\mathrm{X}(\mathrm{Si})$ & $\mathrm{X}(\mathrm{S})$ & $\mathrm{X}(\mathrm{Ca})$ & $\mathrm{X}(\mathrm{Ti})$ & $\mathrm{X}(\mathrm{V})$ & $\mathrm{X}(\mathrm{Cr})$ & $\mathrm{X}(\mathrm{Fe})$ & $\mathrm{X}\left({ }^{56} \mathrm{Ni}\right)$ & $\mathrm{v}_{\text {low }}$ & $\mathrm{v}_{\text {up }}$ \\
\hline 0.000 & 0.170 & 0.000 & 0.080 & 0.150 & 0.020 & 0.005 & 0.005 & 0.020 & 0.020 & 0.070 & 0.460 & 5000 & 5500 \\
\hline 0.000 & 0.200 & 0.000 & 0.080 & 0.120 & 0.020 & 0.005 & 0.005 & 0.020 & 0.020 & 0.070 & 0.460 & 5500 & 6000 \\
\hline 0.000 & 0.230 & 0.000 & 0.080 & 0.100 & 0.020 & 0.005 & 0.005 & 0.015 & 0.015 & 0.070 & 0.460 & 6000 & 6500 \\
\hline 0.000 & 0.270 & 0.000 & 0.080 & 0.080 & 0.020 & 0.005 & 0.005 & 0.015 & 0.015 & 0.070 & 0.440 & 6500 & 7000 \\
\hline 0.000 & 0.310 & 0.000 & 0.080 & 0.070 & 0.015 & 0.005 & 0.000 & 0.010 & 0.010 & 0.070 & 0.430 & 7000 & 7500 \\
\hline 0.000 & 0.330 & 0.000 & 0.080 & 0.070 & 0.015 & 0.005 & 0.000 & 0.000 & 0.000 & 0.070 & 0.430 & 7500 & 8000 \\
\hline 0.000 & 0.350 & 0.005 & 0.080 & 0.030 & 0.010 & 0.005 & 0.000 & 0.000 & 0.000 & 0.070 & 0.440 & 8000 & 8500 \\
\hline 0.000 & 0.470 & 0.030 & 0.070 & 0.000 & 0.000 & 0.005 & 0.000 & 0.000 & 0.000 & 0.055 & 0.370 & 8500 & 9000 \\
\hline 0.060 & 0.520 & 0.050 & 0.030 & 0.000 & 0.000 & 0.004 & 0.000 & 0.000 & 0.000 & 0.040 & 0.300 & 9000 & 9500 \\
\hline 0.150 & 0.620 & 0.050 & 0.000 & 0.000 & 0.000 & 0.002 & 0.000 & 0.000 & 0.000 & 0.020 & 0.160 & 9500 & 10500 \\
\hline 0.200 & 0.700 & 0.050 & 0.000 & 0.000 & 0.000 & 0.000 & 0.000 & 0.000 & 0.000 & 0.000 & 0.000 & 10500 & 11500 \\
\hline 0.250 & 0.700 & 0.050 & 0.000 & 0.000 & 0.000 & 0.000 & 0.000 & 0.000 & 0.000 & 0.000 & 0.000 & 11500 & 12500 \\
\hline 0.250 & 0.700 & 0.050 & 0.000 & 0.000 & 0.000 & 0.000 & 0.000 & 0.000 & 0.000 & 0.000 & 0.000 & 12500 & 13500 \\
\hline
\end{tabular}

Table A5. Mass fractions of chemical elements in velocity layers (between $v_{\text {low }}$ and $v_{\text {up }}$ in $\mathrm{km} \mathrm{s}^{-1}$ ) of the best-fit model for SN $2015 \mathrm{H}$.

\begin{tabular}{|c|c|c|c|c|c|c|c|c|c|c|c|c|c|}
\hline $\mathrm{X}(\mathrm{C})$ & $\mathrm{X}(\mathrm{O})$ & $\mathrm{X}(\mathrm{Na})$ & $\mathrm{X}(\mathrm{Mg})$ & $\mathrm{X}(\mathrm{Si})$ & $\mathrm{X}(\mathrm{S})$ & $\mathrm{X}(\mathrm{Ca})$ & $\mathrm{X}(\mathrm{Ti})$ & $\mathrm{X}(\mathrm{V})$ & $\mathrm{X}(\mathrm{Cr})$ & $\mathrm{X}(\mathrm{Fe})$ & $\mathrm{X}\left({ }^{56} \mathrm{Ni}\right)$ & $\mathrm{v}_{\text {low }}$ & $\mathrm{v}_{\text {up }}$ \\
\hline 0.050 & 0.160 & 0.000 & 0.080 & 0.140 & 0.025 & 0.005 & 0.000 & 0.020 & 0.020 & 0.080 & 0.400 & 5000 & 5500 \\
\hline 0.080 & 0.190 & 0.000 & 0.080 & 0.140 & 0.025 & 0.005 & 0.000 & 0.010 & 0.020 & 0.080 & 0.370 & 5500 & 6000 \\
\hline 0.120 & 0.240 & 0.000 & 0.080 & 0.085 & 0.020 & 0.005 & 0.000 & 0.000 & 0.020 & 0.080 & 0.350 & 6000 & 6500 \\
\hline 0.170 & 0.280 & 0.000 & 0.070 & 0.025 & 0.010 & 0.004 & 0.000 & 0.000 & 0.010 & 0.080 & 0.350 & 6500 & 7000 \\
\hline 0.180 & 0.380 & 0.050 & 0.055 & 0.000 & 0.000 & 0.004 & 0.000 & 0.000 & 0.000 & 0.060 & 0.280 & 7000 & 7500 \\
\hline 0.200 & 0.530 & 0.050 & 0.050 & 0.000 & 0.000 & 0.003 & 0.000 & 0.000 & 0.000 & 0.050 & 0.120 & 7500 & 8000 \\
\hline 0.250 & 0.650 & 0.050 & 0.050 & 0.000 & 0.000 & 0.001 & 0.000 & 0.000 & 0.000 & 0.000 & 0.000 & 8000 & 8500 \\
\hline 0.250 & 0.650 & 0.050 & 0.050 & 0.000 & 0.000 & 0.000 & 0.000 & 0.000 & 0.000 & 0.000 & 0.000 & 8500 & 9000 \\
\hline 0.300 & 0.650 & 0.050 & 0.000 & 0.000 & 0.000 & 0.000 & 0.000 & 0.000 & 0.000 & 0.000 & 0.000 & 9000 & 9500 \\
\hline 0.300 & 0.650 & 0.050 & 0.000 & 0.000 & 0.000 & 0.000 & 0.000 & 0.000 & 0.000 & 0.000 & 0.000 & 9500 & 10000 \\
\hline
\end{tabular}

This paper has been typeset from a $\mathrm{T}_{\mathrm{E}} \mathrm{X} / \mathrm{LT} \mathrm{E} \mathrm{X}$ file prepared by the author. 